APJ, 694, 992

Preprint typeset using LTEX style emulateapj v. 08/22/09

\title{
SPECTRAL ENERGY DISTRIBUTION OF RADIO SOURCES IN NEARBY CLUSTERS OF GALAXIES: IMPLICATIONS FOR SUNYAEV-ZEL'DOVICH EFFECT SURVEYS
}

\author{
Yen-Ting Lin $^{1,2}$, Bruce Partridge ${ }^{3}$, J. C. Pober ${ }^{3}$, Khadija El Bouchefry ${ }^{4,3}$, Sarah Burke ${ }^{5,3}$, \\ Jonathan N. KLein ${ }^{3}$, Joseph W. COISH ${ }^{3}$, And Kevin M. HufFenberger ${ }^{6}$ \\ ApJ, 694, 992
}

\begin{abstract}
To explore the high frequency radio spectra of galaxies in clusters, we used NRAO's Very Large Array at four frequencies, $4.9-43 \mathrm{GHz}$, to observe 139 galaxies in low redshift $(z<0.25)$, X-ray detected, clusters. The clusters were selected from the survey conducted by Ledlow \& Owen, who provided redshifts and $1.4 \mathrm{GHz}$ flux densities for all the radio sources. We find that more than half of the observed sources have steep microwave spectra as generally expected ( $\alpha<-0.5$, in the convention $\left.S \propto \nu^{\alpha}\right)$. However, $60-70 \%$ of the unresolved or barely resolved sources have flat or inverted spectra. Most of these show an upward turn in flux at $\nu>22 \mathrm{GHz}$, implying a higher flux than would be expected from an extrapolation of the lower frequency flux measurements. Our results quantify the need for careful source subtraction in increasingly sensitive measurements of the Sunyaev-Zel'dovich effect in clusters of galaxies (as currently being conducted by, for instance, the Atacama Cosmology Telescope and South Pole Telescope groups).

Subject headings: galaxies: clusters: general - galaxies: active - galaxies: elliptical and lenticular, cD - radio continuum: galaxies
\end{abstract}

\section{INTRODUCTION}

The Sunyaev-Zel'dovich effect (SZE; Sunyaev \& Zel'dovich 1970) is a powerful method for detecting clusters from observations of the cosmic microwave background (CMB). The hot electrons in the intracluster medium inverse-Compton scatter the CMB photons, distorting the $\mathrm{CMB}$ spectrum as seen in the direction of a cluster. Because the SZE is redshift independent and is caused by the presence of dense gas deep within the potential well of dark matter halos, SZE surveys can effectively detect high redshift clusters (see e.g., Carlstrom et al. 2002, for a recent review) and are less confused by large scale structure than optical surveys.

Several microwave background experiments with mJy level sensitivity and 1-10 arcminute beams, including the Atacama Cosmology Telescope (ACT ${ }^{7}$; Fowler et al. 2007), the South Pole Telescope (SPT ${ }^{8}$; Staniszewski et al. 2008; Ruhl et al.|2004), the Arcminute Microkelvin Imager (AMI ${ }^{9}$; Zwart et al. 2008; AMI Collaboration et al. 2006), the Atacama Pathfinder Experiment SZ survey (APEX-SZ ${ }^{10}$ ), and Planck $^{11}$, will yield thousands of SZE clusters in the next few years; in particular, all four ground experiments were already operational in 2007. The data from these surveys will permit

\footnotetext{
${ }^{1}$ Princeton University Observatory, Princeton, NJ 08544; Departamento de Astronomía y Astrofísica, Pontificia Universidad Católica de Chile, Chile

${ }^{2}$ Current address: Institute for the Physics and Mathematics of the Universe, University of Tokyo, Japan; yen-ting.lin@ipmu.jp

${ }^{3}$ Department of Astronomy, Haverford College, Haverford, PA 19041; bpartrid@haverford.edu

${ }^{4}$ Astrophysics and Cosmology Research Unit, University of KwaZuluNatal, South Africa

${ }^{5}$ Swinburne University of Technology, Australia

${ }^{6}$ Jet Propulsion Laboratory, California Institute of Technology, Pasadena, CA 91109; Department of Physics, University of Miami, Coral Gables, FL 33146

7 www.physics.princeton.edu/act/index.html

8 pole.uchicago.edu/

9 Www.mrao.cam.ac.uk/telescopes/ami/index.php

10 bolo.berkeley.edu/apexsz/

11 www.rssd.esa.int/Planck/
}

study of the mass function of clusters over cosmic epochs, a measurement which can elucidate the role of dark energy because structure growth slows during dark energy domination.

As a probe of precision cosmology, a SZE survey has to control its systematics, particularly regarding the correlation between the SZE signal and cluster mass (e.g., Lin \& Mohr 2003). Radio point sources often found at or near cluster centers pose serious challenges in this regard (Carlstrom et al. 2002). Powerful sources can overwhelm the cluster SZE signature (Cooray et al. 1998; Coble et al. 2007), and weaker, unresolved sources can collectively contaminate the SZE signal (Pierpaoli \& Perna 2004). Clusters missed or affected this way would distort the measurements of cosmological parameters from SZE surveys, and it is therefore crucial to estimate the degree of contamination due to radio sources.

Although at low frequencies $(1.4-5 \mathrm{GHz})$ there have been extensive studies of the radio galaxy population in clusters (e.g., Ledlow \& Owen 1996; Miller \& Owen 2001; Morrison \& Owen 2003; Lin \& Mohr 2007), it is not clear at present how these sources behave at the frequencies $(\geq 15$ $\mathrm{GHz})$ and flux levels ( $\sim \mathrm{mJy})$ of on-going SZE surveys. Most of the forecasts for future surveys therefore rely on large extrapolations either in frequency or in flux level, and often both, from existing data (e.g., Toffolatti et al. 1998; Knox et al. 2004; White \& Majumdar 2004; de Zotti et al. 2005; however see Sadler et al. 2008 for recent observations at $95 \mathrm{GHz}$ ). For example, Lin \& Mohr (2007, hereafter LM07) use the observed spectral energy distribution (SED) and spectral index distribution (SID) from 1.4 to $4.85 \mathrm{GHz}$ to estimate SZE survey contamination from the observed $1.4 \mathrm{GHz}$ cluster radio luminosity function. At $150 \mathrm{GHz}$, they estimate that about $10 \%$ of clusters of mass $10^{14}-10^{15} M_{\odot}$ may host AGNs whose total fluxes exceed that of the SZE signal. Although the AGN contribution can be detected and subtracted by combining observations at different frequencies used in a SZE survey, a more critical issue is to be able to quantify the uncertainty about the fraction of clusters being lost or contaminated at few percent level (Lima \& Hu 2005). This extrapolation over two 
orders of magnitude in frequency (i.e., from $1.4 \mathrm{GHz}$ to 150 $\mathrm{GHz}$ ) is highly uncertain, and points out the importance of understanding the actual frequency dependence of these cluster sources.

An extensive follow up of the $15 \mathrm{GHz} 9 \mathrm{C}$ survey from 1.4 to $43 \mathrm{GHz}$ (Bolton et al. 2004) clearly demonstrates that the SED of radio sources is highly non-trivial, but does not focus on cluster radio sources. With sensitive observations toward 89 clusters over $0.1 \leq z<1$, Coble et al. (2007) determine the SID between 1.4 and $28.5 \mathrm{GHz}$. This is a major step toward understanding of the nature of the radio sources in intermediate- to high- $z$ clusters. However, we note that their sample is effectively selected against clusters hosting bright radio sources. Furthermore, only a few of the radio sources are spectroscopically confirmed cluster members. Therefore it is not clear whether their sample is representative of the cluster radio source population as a whole.

Here we present a systematic study of the spectral energy distribution of cluster radio sources from 4.86 to $43.3 \mathrm{GHz}$, conducted with the Very Large Array (VLA). 139 radio galaxies associated with 110 clusters at $z<0.25$ are observed at three or four frequency bands nearly simultaneously, allowing better determination of the spectral shape. Photometric data from the Sloan Digital Sky Survey (SDSS), where available, are used to examine correlations (if any) between the SID/SED and properties of the host galaxy and cluster, such as color, luminosity and clustercentric distance. Our survey improves upon previous studies in several aspects, including the selection of cluster member galaxies based on available redshifts, the large sample size, and the near-simultaneous measurement of fluxes in all four bands.

The plan of the paper is as follows. In $\$ 2$ we describe our cluster and radio galaxy sample. The details of the observations and data reduction are provided in $\$ 3$ and $\$ 4$ respectively. As the angular resolution of the observations at different frequencies is quite different, we pay particular attention in comparing the fluxes in different bands; the procedure is reported in \$5 We present the SED and SID of the sources in $\$ 6$, and the properties of the host galaxies and clusters in $\$ 7$. Based on these new results, we forecast the possible contamination due to radio sources of SZE surveys in $\$ 8$ We conclude by summarizing our main findings and suggesting directions for further work in $\$ 9$

Throughout this paper, we employ a flat $\Lambda \mathrm{CDM}$ cosmological model where $\Omega_{M}=1-\Omega_{\Lambda}=0.3$ and $H_{0}=$ $70 h_{70} \mathrm{kms}^{-1} \mathrm{Mpc}^{-1}$.

\section{CLUSTER AND RADIO GALAXY SAMPLE SELECTION}

Ledlow \& Owen conducted a $1.4 \mathrm{GHz}$ survey of radio galaxies in $\sim 400$ clusters at $z<0.25$ with a limiting sensitivity of $10 \mathrm{mJy}$, and provided extensive redshift measurements for the host galaxies (Ledlow \& Owen 1995, 1996; Owen et al. 1995; Owen \& Ledlow 1997). Their cluster sample was drawn from the Abell catalogs (Abell 1958), and was restricted to area with reddening at $R$-band of less than 0.1 mag. We further limited ourselves to 110 clusters that are detected in X-rays. The main reasons for this requirement are: (1) as our ultimate goal is to make predictions for the radio source contamination in SZE surveys, it is preferable to work with a cluster sample that is selected in a similar fashion as in SZE surveys; and (2) the X-ray emission provides a rough estimate of the cluster mass, which is an important ingredient in our forecast for the SZE surveys. As radio galaxies are rare, to maximize the sample size, we did not set any X-ray flux limit as we compiled our cluster sample. Based on Ledlow \& Owen's redshift catalog, 139 galaxies associated with these clusters were selected as our radio galaxy sample.

We note that Ledlow \& Owen surveyed the galaxies within 0.3 Abell radius of the cluster center (i.e., $\approx 0.64 h_{70}^{-1} \mathrm{Mpc}$ ), irrespective of the size (mass) of the clusters. Given the high concentration in the spatial distribution of radio sources within clusters (LM07), their approach should include the majority of the sources associated with the clusters, thus providing us with a representative initial sample of radio galaxies. Using only sources projected within $40 \%$ of the virial radius does not change the derived SIDs or forecasts on the radio source contamination of the SZE (see $\$ \$ 7 \& 8$ ).

In some cases we detect galaxies not in our initial sample that we could confirm are cluster members on the basis of common redshift (see $\$ 6.1$ ).

\section{OBSERVATIONS}

Measurements in all four spectral bands were made at default VLA frequencies, centered at 43.3, 22.4, 8.5 and 4.9 $\mathrm{GHz}^{12}$. The observations discussed here were made in late October, 2005, with the VLA in a hybrid DnC configuration. In this configuration, the north-south baselines are on average longer than the east-west baselines, and as a consequence the synthesized beam is highly elliptical except for sources observed near the meridian at low elevation. During our runs, several antennas had been removed for repair or refitting; on average we had only 22 available, resulting in a $\sim 20 \%$ reduction in sensitivity from the full array of 27 . The first run, during the night Oct. 23-24, was carried out in mostly cloudy weather with poor atmospheric phase stability. We consequently elected to defer the $43 \mathrm{GHz}$ observations to later runs. The high frequency Q-band observations were concentrated in a short run on Oct. 28 and a much longer run on Oct. 29-30 - the latter in excellent, clear weather. The final short run on the night of Oct. 31 was used to obtain fill-in measurements on sources missed earlier at various frequencies.

\subsection{Calibration}

For all but the Oct. 31 run, our flux density scale was based on 1331+305 (3C286), for which NRAO specifies flux densities of $1.4554,2.5192,5.205$ and $7.485 \mathrm{Jy}$ at $43.3,22.4,8.5$ and $4.9 \mathrm{GHz}$, respectively. 3C286 was not visible during our short run on Oct. 31; for these data we employed 3C48 as the primary flux calibrator, and carefully intercompared the flux densities obtained for sources and secondary calibrators observed in common on this day and earlier ones. In the case of the two highest frequency bands, we employed standard software in the AIPS software package to import a model of the primary calibrators to take account of slight resolution effects in $\mathrm{K}$ and $\mathrm{Q}$ bands.

A variety of secondary (phase) calibrators were employed; we in general selected calibrator sources with reasonably flat spectra so that the same source could be used for observations in all four bands. Calibrators generally were within $\sim 15^{\circ}$ of all of our cluster sources.

Additional information on some instrumental parameters is provided in Table 1 Note that the values for the synthesized

12 Throughout the paper we refer to these frequency bands as $\mathrm{Q}, \mathrm{K}, \mathrm{X}$, and $\mathrm{C}$ bands, respectively. Note that the $1.4 \mathrm{GHz}$ channel is denoted as the $\mathrm{L}$ band. 
beam shape are approximate, since the beam geometry depends on the declination and hour angle of the source.

\subsection{Fast Switching}

In the case of the $43 \mathrm{GHz}$ observations, we employed fast switching between the source of interest and a nearby phase calibrator source. The integration times on source and calibrator were set to be approximately equal to or less than the atmospheric phase coherence time at $43 \mathrm{GHz}$. Rather than adjusting these integration times on the fly, we set them to be $100 \mathrm{sec}$ on sources between calibrations, and $40 \mathrm{sec}$ on nearby calibrators. For each galaxy observed, this cycle was repeated 3 times.

\section{DATA REDUCTION, ANALYSIS, AND IMAGING}

The raw amplitude and phase data are flagged for shadowing of one antenna by another, interference, noisy correlators, weak antennas, and so on. In general, this flagging process removes only a few percent of the raw data. When data from the available antennas in the array are combined, the data are weighted by the inverse of the variance in the average signal.

Each source at each of the four frequencies is imaged using standard NRAO procedures in the AIPS software package. In forming the images, the pixel or cell size initially employed is $0.1^{\prime \prime}, 0.2^{\prime \prime}, 0.6^{\prime \prime}$ and $1.0^{\prime \prime}$ at $43.3,22.5,8.5$, and $4.9 \mathrm{GHz}$, respectively. These values allow complete sampling of the synthesized beam even along its minor axis. In all cases, we make $1024^{2}$ images. The raw images are lightly cleaned of side lobes ( $\sim 200$ iterations) again using standard NRAO procedures in AIPS. In most cases, when a source or sources are evident in the raw image, we clean first in a small area containing the source(s), then lightly clean the entire $1024^{2}$ pixel image. If no source is evident in the initial image, we simply clean lightly over the entire area. We have experimented with different levels of cleaning, and found no significant change in the flux densities of sources.

\subsection{Flux Density of Unresolved or Barely Resolved Sources}

Flux densities of evident, and unresolved or barely resolved, sources are determined by fitting a two-dimensional Gaussian to each image, using a standard process in AIPS (specifically, IMFIT). We report the integrated flux for each source. When no source is evident at or near the specified position, we compare $4 \sigma$ ( $\sigma$ is the local noise rms) to $2 \sigma$ added onto the brightest flux per beam near the image center (within 50 pixels), and present the larger of the two as an upper limit.

\section{EFFECTS OF RESOLUTION}

As expected, many cluster radio sources show evident, resolved, structure at one or more of our observing frequencies. For resolved or irregular sources it is more difficult to obtain accurate fluxes; more importantly, the flux densities of resolved and complex sources are difficult to compare at different frequencies. For instance, the lobes of some of the classical FRII radio sources in our list are well delineated at $4.9 \mathrm{GHz}$, but only isolated hot spots in the lobes are visible at higher frequencies. In addition, the angular resolution of the VLA synthesized beam in the DnC configuration varies from $\sim 2^{\prime \prime}$ to $\sim 13^{\prime \prime}$ depending on frequency; much of the flux of extended sources is resolved out at higher frequencies. When flux is resolved out, only lower limits can be placed on spectral indices.

\subsection{Tapered $43 \mathrm{GHz}$ Images}

Since we are most interested in the highest frequencies, 22 and $43 \mathrm{GHz}$, flux densities and the $22-43 \mathrm{GHz}$ spectral index, we convolve our $43 \mathrm{GHz}$ images with an elliptical Gaussian weighting profile to broaden the synthesized beam to match approximately the larger size of the synthesized beam at 22 $\mathrm{GHz}$. This is done by applying a Gaussian weighting function to the $u-v$ data before imaging, again using a standard procedure in the AIPS task IMAGR. A $u-v$ taper of $45 k \lambda$ and $135 \mathrm{k} \lambda$ provides a good overall match to the $22 \mathrm{GHz}$ beam. For these tapered images we employ $0.2^{\prime \prime}$ cells, as for the 22 $\mathrm{GHz}$ images. By approximately matching the 22 and $43 \mathrm{GHz}$ synthesized beams, we eliminate or reduce the problem of resolution and are able to compare fluxes from matched areas of the sky. Thus our 22-43 GHz spectral indices are unbiased values.

Unless otherwise noted, all flux densities at $43 \mathrm{GHz}$ are derived from these tapered images.

Because of the larger frequency ratio between 8.5 and 22 $\mathrm{GHz}$, tapering the $22 \mathrm{GHz}$ images to match the $8.5 \mathrm{GHz}$ synthesized beam produces very noisy images (much of the $u-v$ data was strongly down-weighted), so we elect not to taper the $22 \mathrm{GHz}$ images; see $\$ 6.4$ for further details. Hence spectral indices based on fluxes at 8.5 (or 4.9) GHz are lower limits, as noted above.

\subsection{Flux Density of Resolved or Irregular Sources}

In the case of irregularly shaped or clearly extended sources, we estimate the flux density within a rectangular region including all of the visible emission. These are figures cited in Table 5 (see $\$ 6$ ). Relatively few of the 22 and $43 \mathrm{GHz}$ sources are complex enough to require this treatment.

\section{OBSERVATIONAL RESULTS}

We have observed 139 galaxies, and detected 136 in at least one band. The three that show no sign of a source at any of the frequencies are 0053-102B, 1108+410A, and 1657+325B. These three are not included in our analysis. We note that the first and last of these three are weak $21 \mathrm{~cm}$ sources in Ledlow \& Owen's catalog. On the other hand, 1108+410A has a flux of $116 \mathrm{mJy}$ in their catalog, but is very extended. In our 4.9 $\mathrm{GHz}$ image, we see faint traces of a source, but it is almost entirely resolved out even at $\sim 12^{\prime \prime}$ resolution.

For 111 galaxies we are able to measure flux in at least three bands. This subsample will allow better determination of the spectral shape, and will be the focus of this section. Some of these galaxies have multiple components, and in total we detect 140 radio sources associated with them. Table 2 records the detection statistics of our sources. In Table 5 we provide the available flux density measurements of all the sources. Most blank entries in Table 5 are for background sources (see $\$ 6.1$ far enough from the image centers so that they are not contained within the primary beams at the two higher frequencies we employed. In other cases, our runs on a particular source at a particular frequency were spoiled by weather or lost for other reasons. In a smaller number of cases, including for instance $0816+526$, sources seen independently at the higher frequencies were blended at $5 \mathrm{GHz}$, so that it was not possible to determine accurate flux densities at that frequency.

\section{1. "Background" Sources}

In many of our images, especially those at low frequencies with their correspondingly larger solid angle, we by chance 
TABLE 1

INSTRUMENTAL PARAMETERS

\begin{tabular}{ccccc}
\hline \hline $\begin{array}{c}\text { Frequency } \\
(\mathrm{GHz})\end{array}$ & $\begin{array}{c}\text { Integration Time } \\
\text { (seconds) }\end{array}$ & $\begin{array}{c}\text { Typical Image Sensitivity } \\
(\mathrm{mJy})\end{array}$ & $\begin{array}{c}\text { Image Pixel Size } \\
(\operatorname{arcsec})\end{array}$ & $\begin{array}{c}\text { Synthesized Beam } \\
\text { (approx.) }\end{array}$ \\
\hline 4.86 & 80 & 2.0 & 1.0 & $8^{\prime \prime} \times 13^{\prime \prime}$ \\
8.46 & 50 & 0.5 & 0.6 & $4^{\prime \prime} \times 8^{\prime \prime}$ \\
22.46 & 120 & 1.0 & 0.2 & $2^{\prime \prime} \times 3^{\prime \prime}$ \\
43.34 & $\sim 300^{\mathrm{a}}$ & 0.8 & $0.2^{\mathrm{b}}$ & $2^{\prime \prime} \times 3^{\prime \prime \mathrm{b}}$ \\
\hline
\end{tabular}

a fast switching employed (see $\sqrt[53.2]{3}$.

$\mathrm{b}$ for tapered images (see $\$ 5.1$.
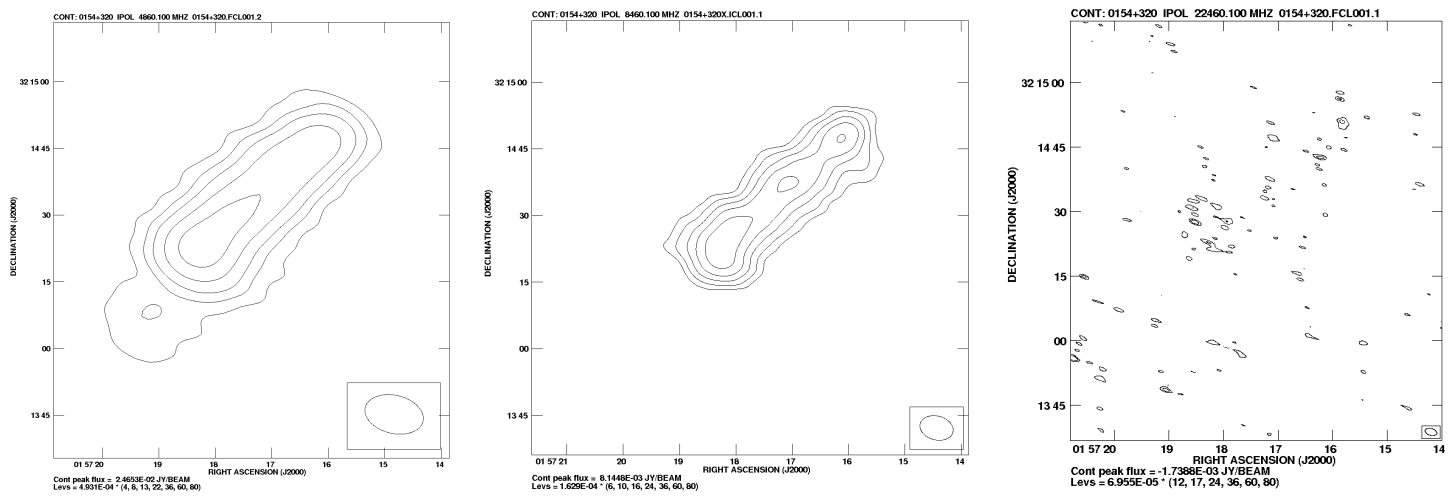

FIG. 1. - Left to right: a source $(0154+320)$ imaged at $\mathrm{C}, \mathrm{X}$ and $\mathrm{K}$ bands, showing the loss of flux due to resolution; beam profiles at each frequency are shown in the small box on the lower right of each panel. Contours are selected to reveal the main source properties.

TABLE 2

DETECTION STATISTICS

\begin{tabular}{ccccc}
\hline \hline & \multicolumn{2}{c}{ all morphologies } & \multicolumn{2}{c}{ core/point-like } \\
\cline { 2 - 5 } detection $^{\mathrm{a}}$ & components & galaxies & components & galaxies \\
\hline all 4 & 87 & 75 & 57 & 57 \\
$\geq 3$ & 140 & 111 & 73 & 73 \\
$\geq 2$ & 185 & 133 & 83 & 83 \\
$\geq 1$ & 192 & 136 & 86 & 85 \\
\hline total & $195^{\mathrm{b}}$ & $139^{\mathrm{b}}$ & 86 & 85 \\
\hline
\end{tabular}

${ }^{a}$ number of bands in which the sources are detected.

b three sources detected at $1.4 \mathrm{GHz}$ by Owen \& Ledlow (1997) were not detected at any frequency in our VLA observations.

detect sources at a distance from the center of the field (or pointing position). Since our target galaxies are all at low redshift, these peripheral sources are presumably mostly background radio sources. We exclude from our analysis of the statistical properties of cluster radio galaxies all such "background" sources. Four of these "background" sources, however, have catalogued redshifts which show they are cluster members. These 4 are added to our sample of cluster galaxies in the subsequent analysis.

\subsection{Overall Properties of Cluster Radio Galaxies}

We now focus on the 111 cluster radio galaxies for which the spectral shape can be reliably traced, since we have measurements at $\geq 3$ frequencies. At the lowest frequency, 4.9 $\mathrm{GHz}$, virtually all of the sources have complex structure. In $\sim 75 \%$ of the sources, a clear core or small, barely resolved jet is visible. Even in these cases however, there is generally additional extended emission. In other sources, the cores or other resolved or barely resolved structures visible in
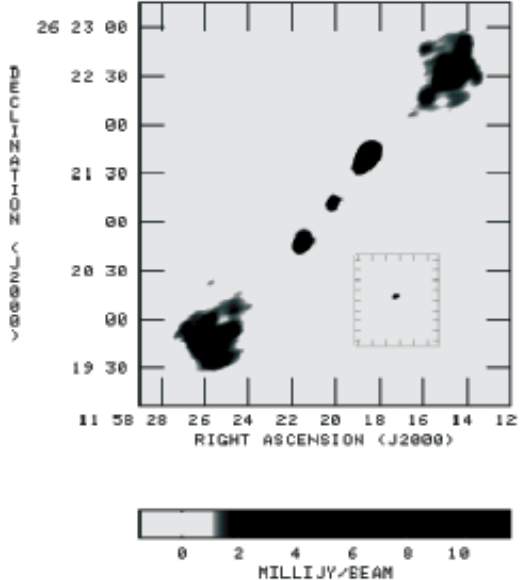

FIG. 2.- Main figure: the source $1155+266$ imaged at $8.5 \mathrm{GHz}$. Insert (to same scale): the same source at $22 \mathrm{GHz}$, showing no evidence for emission from the lobes; the core remains.

higher frequency, higher resolution, images are unresolved or merged with diffuse structure in the $4.86 \mathrm{GHz}$ images. An example is shown in Fig. 1. This makes it difficult to isolate the cores at our lowest frequency, and to determine their flux densities for comparison with measurements at higher frequencies. In some cases, our best option is to compare the 4.9 $\mathrm{GHz}$ flux of an entire source with the sum of the flux densities of its components at the next highest frequency.

At the next highest frequency, $8.5 \mathrm{GHz}$, with a $4^{\prime \prime} \times 8^{\prime \prime}$ beam, cores and jets are more frequently resolved and isolated. On the other hand, we are resolving out some of the flux of extended features seen in the lower resolution $4.9 \mathrm{GHz}$ im- 
age. As already noted, that means that the $8.5 \mathrm{GHz}$ flux densities may be underestimated for large, extended sources and hence our calculated values of the 4.9-8.5 GHz spectral index are generally lower limits. On the other hand, the flux density determination for isolated, barely resolved cores and jets are more accurate and less influenced by background emission than is the case at $4.9 \mathrm{GHz}$.

At the two highest frequencies, because of the higher resolution, most of the extended structure seen at the two lower frequencies is resolved out and barely visible or not apparent (see Fig. 2). As noted in $\$ 5.1$ above, we convolve the 43 $\mathrm{GHz}$ images to produce a synthesized beam matching that at $22 \mathrm{GHz}$. Thus we can fairly intercompare flux densities at the two higher frequencies, but it remains the case that spectral indices involving flux densities at either of the two lower frequencies will be lower limits.

\subsection{SEDs of Cores and Other Point-like or Barely Resolved Sources}

The very different resolution of our images at different frequencies makes it difficult to compare flux densities directly, and hence to determine SEDs, especially for complex or resolved sources. We therefore elect to concentrate on unresolved or barely resolved sources or the obvious cores within more complex structure. These sources are flagged in column 4 of Table 5 with a "C" indicating a well defined core or "P" indicating an unresolved or barely resolved "point-like" source. We are not claiming that these sources are necessarily unresolved at $\sim 1^{\prime \prime}$ scale, but rather that they are sufficiently isolated and regular in appearance that accurate flux densities can be obtained.

Of the 140 cluster sources for which the determination of a SED is possible, 73 or $52 \%$ are either point-like or barely resolved in one or more of our three highest frequency images or have a clearly identifiable core at one or more of these same frequencies.

\subsection{SEDs of Extended Sources}

Because of the resolution effects discussed in $\$ 5.1$ our SEDs and spectral indices for extended sources are less certain. In general, as expected (e.g., de Young 2002), the lobes and diffuse structure show steep spectral indices. In a few cases, as an experiment, we convolve the $8.5 \mathrm{GHz}$ images to match the resolution of the $4.9 \mathrm{GHz}$ images to allow direct comparison of fluxes. The results are shown in Table 3 From the table, it is clear that in these sample cases, at least, resolution is not significantly affecting the $8.5 \mathrm{GHz}$ fluxes in major ways, except for clearly resolved sources.

If only emission from lobes and extended structure were involved, the generally steep spectra would ensure that most cluster radio sources would present minimal problems for SZE measurements carried out at frequencies above, say, 90 GHz. The second-to-last column of Table 5 lists estimated 90 $\mathrm{GHz}$ flux densities based on the $\mathrm{X}$ band flux, assuming that the spectral index between 4.9 and $8.5 \mathrm{GHz}$ can be directly extrapolated to $90 \mathrm{GHz}$. The last column of the Table is the estimate based on the $\mathrm{Q}$ band flux where available, using the spectral index between 22 and $43 \mathrm{GHz}$. Note the frequent substantial differences in extrapolated flux.

\subsection{SEDs of Cores}

However, we find that the cores and other unresolved or barely resolved structures generally have flatter spectra, and

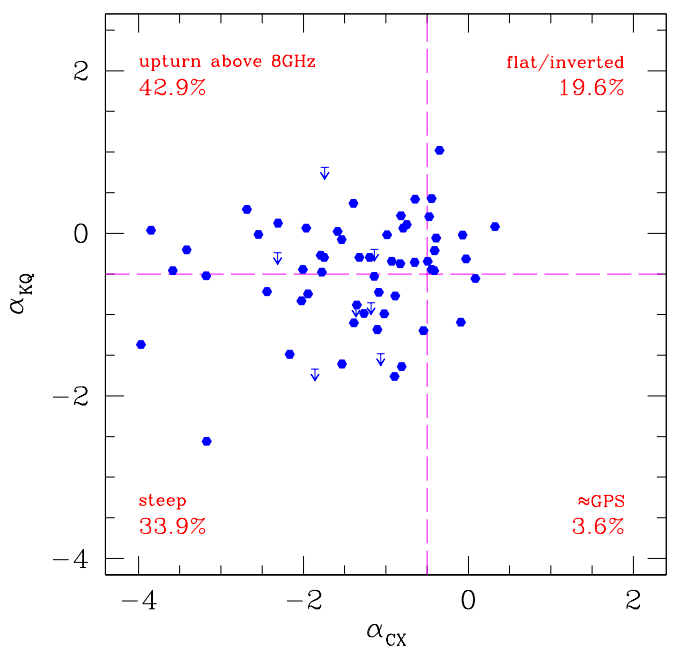

FIG. 3.- The distribution of the spectral indices provides a way to quantify the relative proportions of different spectral shapes, as indicated in the four quadrants. We list the percentage of each type of spectral shape in the corresponding quadrants. Note that a large fraction of sources exhibit curvature in their spectra (e.g., the "upturn" type).

in particular that many sources exhibit a substantial change in spectral index at frequencies above $22 \mathrm{GHz}$. This means that cluster radio sources may present a larger problem for sensitive SZE measurements than might be expected from the extrapolation of low frequency measurements (e.g., LM07). For that reason, as well as because of the difficulty of obtaining the fluxes of extended sources, we concentrate on cores and other unresolved or barely resolved components. We will focus on the 73 sources that are detected in three or more bands with these morphologies. It is important to recall, however, that even for these relatively compact and uncomplicated sources, resolution effects may cause us to miss some of the flux. Since we use tapered $\mathrm{Q}$ band flux densities, $\alpha_{K Q}$ is nominally unaffected, but resolution may affect the spectral indices at lower frequencies.

An efficient way to quantify the distribution of the spectral shapes is through the "two-color" plot (Sadler et al. 2006), shown in Fig. 3. where the spectral index between the C \& X bands $\left(\alpha_{C X}\right)$ is plotted for 57 core/point-like sources against that between the $\mathrm{K} \& \mathrm{Q}$ bands $\left(\alpha_{K Q}\right)$. Throughout our analysis, we adopt the notation for the spectral index such that a power-law spectrum is described as $S_{\nu} \propto \nu^{\alpha}$. Following the common practice of referring to sources with $\alpha<-0.5$ as "steep", and "flat/inverted" otherwise, the $\alpha_{C X}-\alpha_{K Q}$ space is divided into four quadrants, as delineated by the two dashed lines in Fig. 3 Starting from the first quadrant (upper-right corner) and going counterclockwise, the quadrants contain sources with flat/inverted spectra, with spectra that turn flat above $8 \mathrm{GHz}$, with steep spectra, and with spectra that peak around 10 or so $\mathrm{GHz}$ [which we refer to as "approximately gigahertz peaked spectrum" ( $\approx$ GPS) sources], respectively. The relative proportions of these types of spectral shape are shown in Fig. 3 We can also see that $\sim 84 \%$ show a more positive high frequency spectral index than low frequency spectral index, that is a flattening at frequencies above $8 \mathrm{GHz}$ or so. Only about one third of the sources have steep spectra from 4.9 to $43 \mathrm{GHz}$. The lack of correlations of the data points clearly suggests that the spectral shape of the core/point-like 
TABLE 3

EFFECT OF TAPERING ON X-BAND FLUX MEASUREMENTS

\begin{tabular}{|c|c|c|c|c|c|}
\hline Source & Extended & $\begin{array}{l}\text { X Flux (un-tapered) } \\
(\mathrm{mJy})\end{array}$ & $\begin{array}{l}\text { X Flux (tapered) } \\
(\mathrm{mJy})\end{array}$ & $\begin{array}{l}\text { C Flux } \\
(\mathrm{mJy})\end{array}$ & $\alpha_{C X}$ (tapered) \\
\hline 0036-226B & & $59.850 \pm 0.430$ & $61.770 \pm 0.520$ & $74.330 \pm 0.733$ & -0.334 \\
\hline $0037+292$ & & $2.910 \pm 0.320$ & $3.110 \pm 0.340$ & $6.200 \pm 0.500$ & -1.245 \\
\hline 0039-095B & & $7.040 \pm 0.240$ & $7.230 \pm 0.290$ & $13.260 \pm 0.644$ & -1.094 \\
\hline 0100-221A & & $4.495 \pm 0.520$ & $3.180 \pm 0.260$ & $6.110 \pm 0.570$ & -1.178 \\
\hline $0119+193(1)$ & & $8.785 \pm 0.320$ & $8.480 \pm 0.300$ & $11.880 \pm 0.610$ & -0.608 \\
\hline $0124+189$ & Yes & $75.440 \pm 1.910$ & $132.900 \pm 2.830$ & $310.000 \pm 4.260$ & -1.528 \\
\hline $0139+073 \mathrm{~A}$ & & $4.898 \pm 0.230$ & $4.710 \pm 0.320$ & $6.270 \pm 1.040$ & -0.516 \\
\hline $0909+161$ & & $10.430 \pm 0.340$ & $11.380 \pm 0.410$ & $16.410 \pm 0.910$ & -0.660 \\
\hline $1058+107$ & & $7.935 \pm 0.290$ & $9.920 \pm 0.480$ & $9.957 \pm 0.390$ & -0.007 \\
\hline $1130+148$ & & $8.820 \pm 0.390$ & $9.630 \pm 0.560$ & $13.680 \pm 0.410$ & -0.633 \\
\hline $1132+492$ & & $31.860 \pm 0.740$ & $34.640 \pm 0.900$ & $30.380 \pm 0.830$ & 0.237 \\
\hline $1201+282$ & & $1.870 \pm 0.430$ & $1.680 \pm 0.550$ & $2.930 \pm 0.310$ & -1.003 \\
\hline $1301+195$ & & $13.700 \pm 0.270$ & $12.790 \pm 0.290$ & $22.510 \pm 0.280$ & -1.020 \\
\hline $1433+553$ & Yes & $16.810 \pm 0.550$ & $21.870 \pm 0.710$ & $73.920 \pm 1.020$ & -2.197 \\
\hline $1435+249$ & & $9.210 \pm 0.390$ & $10.160 \pm 0.500$ & $11.800 \pm 0.560$ & -0.270 \\
\hline $2228-087$ & & $10.480 \pm 0.312$ & $11.190 \pm 0.320$ & $10.893 \pm 0.670$ & 0.049 \\
\hline $2333+208$ & & $6.601 \pm 0.270$ & $7.680 \pm 0.290$ & $11.410 \pm 0.330$ & -0.714 \\
\hline $2348+058$ & & $3.022 \pm 0.240$ & $3.400 \pm 0.250$ & $8.490 \pm 0.290$ & -1.651 \\
\hline
\end{tabular}

TABLE 4

MEAN SPECTRAL INDICES

\begin{tabular}{ccc}
\hline \hline Bands & All sources & Cores/point-like \\
\hline $5-8$ & $-1.64 \pm 0.10$ & $-1.31 \pm 0.10$ \\
$8-22$ & $-1.20 \pm 0.07$ & $-0.88 \pm 0.09$ \\
$22-43$ & $-0.98 \pm 0.11$ & $-0.62 \pm 0.10$ \\
\hline
\end{tabular}

sources is non-trivial.

If we include all 75 sources irrespective of their morphology, the relative proportions of the four quadrants become $13.1 \%$ (flat/inverted), $35.7 \%$ (upturn), $48.8 \%$ (steep), and $2.4 \%(\approx \mathrm{GPS})$.

With $\sim 100$ sources detected at $18 \mathrm{GHz}$ with the Australian Telescope Compact Array (ATCA), Sadler et al. (2006) study the distribution of the spectral shapes with the two-color plot, where their low and high frequency indices are based on 0.8 \& $5 \mathrm{GHz}$, and $8 \& 18 \mathrm{GHz}$ fluxes, respectively. We note that they separate the flat/inverted sources from steep ones at $\alpha=0$; adopting the same definition, we find that the great majority of our sources become steep (73\%) and upturn (23\%). This seems to suggest that our cluster sources exhibit steeper spectra than theirs. However, the fact that the two samples are selected at very different frequencies (1.4 v.s. $18 \mathrm{GHz})$ needs to be taken into consideration. In addition, although the majority of their sources are likely QSOs and BL Lac objects, most of them lack redshift information, which makes it difficult to make a fair comparison (e.g., the nature of the sources and their environments, as well as possible cosmological evolution). Nevertheless, we agree with their conclusion that extrapolation of fluxes to high frequencies (e.g., $\gtrsim 10$ $\mathrm{GHz}$ ) based on low frequency observations is not reliable.

\subsection{Spectral Index Distribution}

Here we quantify the spectral index distribution in the 5-8, $8-22$, and $22-43 \mathrm{GHz}$ bands. An important aspect in estimating the SIDs is to deal with sources for which only an upper limit in flux in one of the bands is available, which leads to upper or lower limits of the spectral index. To accommodate such cases, we calculate the distribution with the ASURV package (Feigelson \& Nelson 1985; Isobe et al. 1986), which is based on survival statistics, a branch of statistics developed

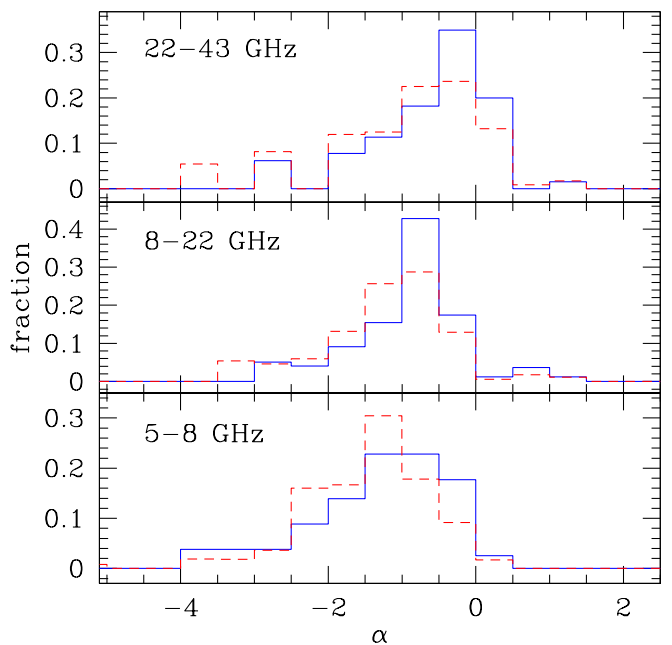

FIG. 4.- The spectral index distribution in three frequency bands. The solid histogram is the result when only sources with core/point-like morphology are used. The dashed histogram is obtained when all sources are included.

in actuarial estimates of human survival and mortality (see e.g., Feigelson \& Nelson 1985 for a review). The resulting SIDs are shown in Fig. 4, where the solid histogram is for sources with core/point-like morphology, and the dashed histogram is for all sources. We record the mean values of the indices in Table 4 . As expected, the SIDs based on all the sources have a mean that is more negative.

A recent study presents the spectral indices between 1.4 \& $28.5 \mathrm{GHz}$ for 95 probable cluster radio sources (Coble et al. 2007). They find that the mean of the index is $\sim-0.7$. Because of the differences between the beam size of the $1.4 \mathrm{GHz}$ observations made by Ledlow \& Owen (1996) and ours, we do not attempt to calculate an analog to their spectral index (e.g., $\left.\alpha_{L K}\right)$. Furthermore, as the spectral shape tends to be complicated, it is not clear how much predictive power an index spanning such a wide range in frequency would have.

\section{CORRELATION OF SPECTRAL INDICES AND PROPERTIES OF THE HOST GALAXIES AND CLUSTERS}




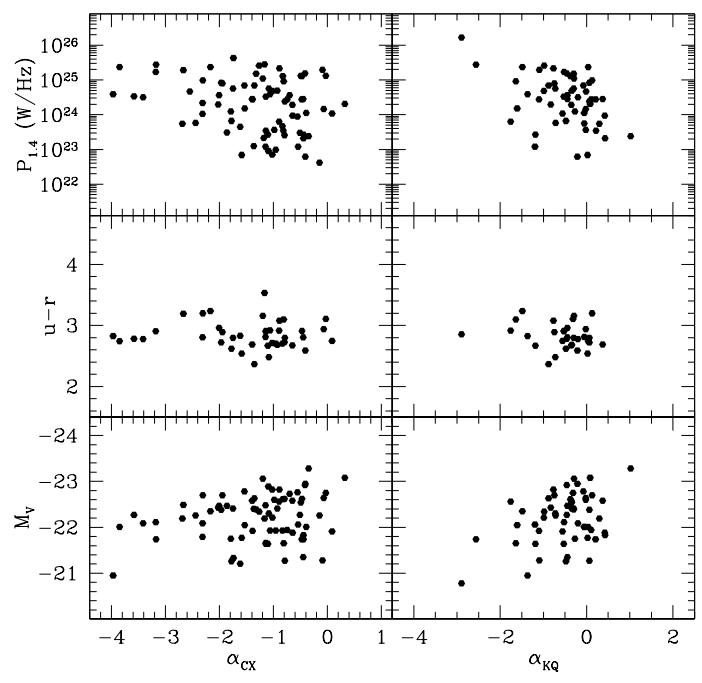

FIG. 5.- Distribution of the spectral indices with respect to properties of host galaxies. We consider the absolute optical (V-band) magnitude, the optical color $(u-r)$, and the radio power at $1.4 \mathrm{GHz}$ of the hosts. No apparent correlation is found.

Next we examine if there is any correlation between the spectral indices (from sources of core/point-like morphology) and the properties of the host galaxies or of the clusters. In particular, we consider the optical and radio luminosities, as well as the optical color, of the host galaxies. As for the cluster-related properties, we look at the mass and redshift of the clusters, and the projected radial distance to the cluster center, which is determined from the emission peak of the intracluster gas.

In Fig. 5 we show scatter plots between $\alpha_{C X} / \alpha_{K O}$ and the galaxy properties. Fig.6 is the corresponding plot for the cluster properties. A few points are worth commenting on both Figures. First, we note that the host galaxies are of moderate optical luminosity (recall that $M_{*}=-20.8$ in the V-band), and are red in color $(u-r>2.2)$. Inspecting the optical images of the host galaxies from SDSS confirms that most of the galaxies are early type, of elliptical morphology. The distribution of the $1.4 \mathrm{GHz}$ luminosities $\left(P_{1.4}\right)$ suggests that these galaxies are likely FRI-type radio-loud AGNs.

The cluster mass $M_{200}$ is estimated from the X-ray luminosity $\left(L_{X}\right)$-virial mass relation (Reiprich \& Böhringer 2002). $M_{200}$ is defined as the mass enclosed by $r_{200}$, a radius within which the mean overdensity is 200 times the critical density. Because of the scatter in the $L_{X}-M_{200}$ relation, our mass estimate is only accurate to $\lesssim 50 \%$ (Reiprich \& Böhringer 2002). Nevertheless, it is shown that $L_{X}$ is a unbiased mass indicator (Reiprich 2006). As our main purpose is to find correlations with the cluster mass, $L_{X}$ should suffice as a proxy for mass. For each radio source, we normalize its clustercentric distance by $r_{200}$, to account for the difference in cluster mass. As Fig. 6) buggests, our clusters span a range $>20$ in mass. The majority of the sources are concentrated toward the cluster center, which confirms several earlier findings (e.g., Morrison \& Owen 2003; LM07).

It is interesting to see that there appears to be no strong correlations between the spectral indices and the host galaxies/clusters. The Spearman's rank correlation coefficients for all cases we examine are between -0.1 and -0.34 , indicating no significant correlations. The pair of properties that shows

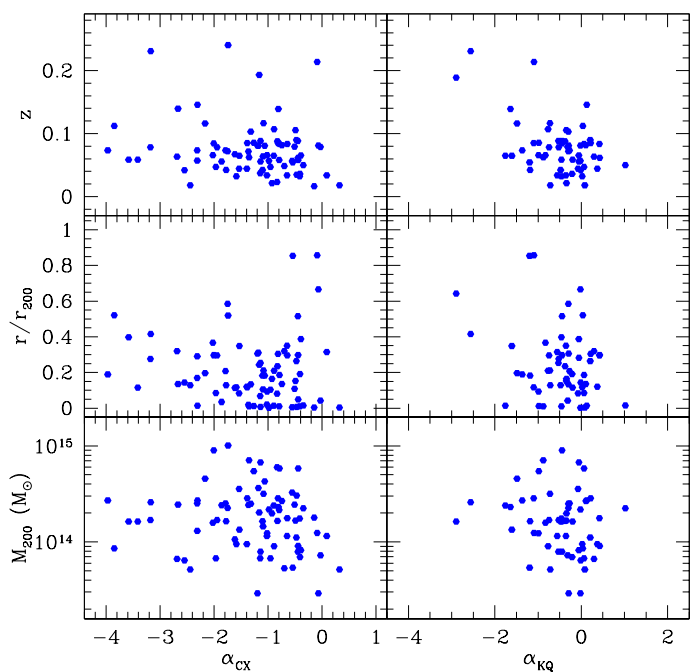

FIG. 6.- Distribution of the spectral indices with respect to properties of host clusters. We examine the mass of the clusters, the distance to the cluster center (normalized by the virial radius of the cluster, $r_{200}$ ), and the redshift As expected, radio sources concentrate towards cluster center. As in Fig. 5 we do not find significant correlations.

the strongest correlation is that between $\alpha_{K Q}$ and $P_{1.4}$ (correlation coefficient $=-0.34$ ). But this is mainly driven by a couple of sources that have the most negative spectral index.

Coble et al. (2007) do not find any difference between the spectral indices for sources in the inner and those in the outer parts of the clusters, suggesting lack of correlation with clustercentric distance, which is consistent with our finding here. Considering the fact that spatial distribution of the lowpower radio galaxies is very concentrated towards cluster center (LM07), this seems to suggest that although being near the center of massive halos increases the probability of accretion onto the supermassive blackholes (e.g., high gas density or/and pressure from the intracluster medium), the resulting emission is dominated by the small scale physics of the nucleus rather than by the cluster environment.

\section{IMPLICATIONS FOR SZE SURVEYS}

The main motivation to conduct the present study is to characterize the SED/SID of radio sources associated with galaxy clusters, which can be used to assess their effect on the detection and characterization of clusters through the SZE. Simply put, the SIDs can be used to extrapolate the observed radio luminosity function (RLF) at low frequencies to the frequency of an SZE experiment, which in turn provides an estimate of the abundance of radio sources.

Our approach is similar to that of LM07, and we refer the reader to that paper for more details ( $\$ 7$ therein). We will only provide an overview of the method here. The basic idea is to use the (observed) RLF within clusters and groups to predict the number and flux of radio sources expected in massive halos of given mass and redshift. Specifically, the RLF gives the number density of radio sources which, when multiplied by the volume of the halo, becomes the number of sources expected. One can draw (Poisson) random numbers from it, and assign radio luminosities according to the RLF. On the other hand, given the mass and redshift of a halo, one can predict its SZE signal, which can be compared with the total fluxes from the radio sources. By repeating this procedure for a large number of halos of the same mass and redshift, one 


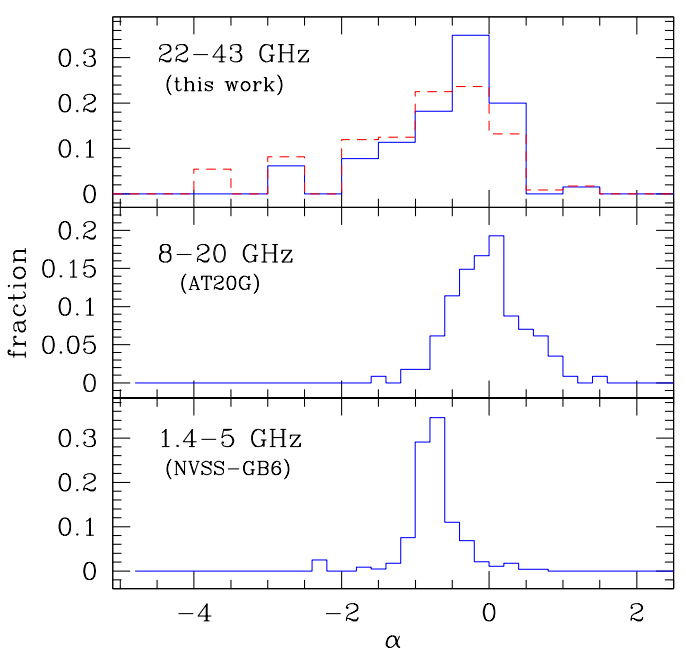

FIG. 7.- The spectral index distribution in three frequency bands. Top: the SID in the $22-43 \mathrm{GHz}$ band as determined from our VLA data; this is identical to that shown in the top panel of Fig. 4 Middle: the $8-20 \mathrm{GHz}$ SID determined from the AT20G survey, using data presented in Sadler et al. (2006). The mean of the distribution is $\alpha_{X K, A T 20 G}=-0.028 \pm 0.046$. Bottom: the $1.4-5 \mathrm{GHz}$ SID based on data from the NVSS and GB6 surveys. The mean is $\alpha_{L C, N V S S / G B 6}=-0.754 \pm 0.024$. The details of the construction of $\operatorname{SID}(8-20$, AT20G) and SID(1.4-5, NVSS/GB6) are described in the Appendix. Compared to the middle and bottom panels in Fig.4 the SIDs in this figure are more positive. In particular, as AT20G is a $20 \mathrm{GHz}$-selected survey, the resulting SID is biased towards flat-spectrum sources.

produces a radio galaxy catalog in a Monte Carlo fashion, and can determine the fraction of clusters that are significantly affected by the radio sources they host. In $\$ 8.1$ we describe our scheme for extrapolating the RLFs, and in $\$ 8.2$ we present our estimates of the contamination of the SZE due to cluster radio sources.

\subsection{Extrapolation of the Radio Luminosity Function}

The main ingredients in our method include: the $1.4 \mathrm{GHz}$ RLF of radio sources residing in massive halos, the SIDs between several frequencies, and a model for the redshift evolution of the RLF. The 1.4 GHz RLF in units of space density is measured in LM07, and we give in $\$ 7$ the distributions for $\alpha_{C X}, \alpha_{X K}$, and $\alpha_{K Q}$. For SZE surveys operating at $\sim 150 \mathrm{GHz}$ (e.g., ACT and SPT), for low redshift sources, our modeling requires a factor of $\sim 4$ extrapolation of the radio spectra in frequency (i.e., from $43 \mathrm{GHz}$ ), which represents a dramatic improvement from that of LM07, which adapted an SID from 1.4 and $4.85 \mathrm{GHz}$ measurements. However, because of the mismatch between the angular resolutions in our images at $\mathrm{C}, \mathrm{X}$, and $\mathrm{K}$ bands, the spectral indices we determine between these bands may be lower limits, and thus the SIDs of $\alpha_{C X}$ and $\alpha_{X K}$ may be biased towards negative values. To assess the effects of the choices of SIDs, we will utilize other data sets to determine the SIDs at $\nu \lesssim 20 \mathrm{GHz}$. To this end, we combine the catalogs from the NVSS (Condon et al. 1998) and GB6 (Gregory et al. 1996) surveys to measure the $1.4-4.85 \mathrm{GHz}$ SID, and use the results from the AT20G survey (Sadler et al. 2006) to measure the $8.5-20 \mathrm{GHz}$ SID. We describe the construction of the matched NVSS/GB6 sample, as well as the AT20G data, in the Appendix. As the beam sizes of both NVSS and GB6 surveys are large $\left(45^{\prime \prime}\right.$ and $3.5^{\prime}$, respectively), the flux, and in turn the spectral index $\alpha_{L C}$ measurements, should be reliable except for very extended sources. On the other hand, the AT20G survey selects sources at $20 \mathrm{GHz}$, and the resulting sample would be biased towards flat-spectrum sources. These SIDs are shown in the middle and lower panels in Fig. 7 The mean values of $\alpha_{L C, N V S S / G B 6}$ and $\alpha_{X K, A T 20 G}$ are $-0.754 \pm 0.024$ and $-0.028 \pm 0.046$, respectively. Using the SID(1.4-4.85, NVSS/GB6) and SID(8-20, AT20G) rather than those presented in $\$ 7$ will produce extrapolated RLFs with higher amplitude (i.e., more radio sources), resulting in higher estimates of the contamination of SZE signals. We caution that the SIDs from NVSS/GB6 and AT20G are not limited to radio sources in groups and clusters ${ }^{13}$ (although the NVSS/GB6 sources are constrained to be at $z<0.4$ ). However, incorporating these SIDs allows us to explore the degree of AGN contamination of the SZE to a fuller extent.

LM07 measure the $1.4 \mathrm{GHz}$ RLF for cluster radio sources. We transform that RLF to higher frequencies by convolving it with the spectral index distribution via (LM07)

$$
\phi_{2}\left(\log P_{2}\right)=\int \phi_{1}\left(\log P_{2}+\alpha \log \left(\nu_{1} / \nu_{2}\right)\right) f\left(\alpha_{12}\right) d \alpha_{12}
$$

where $\phi \equiv d n / d \log P$ is the RLF, and subscripts refer to two frequencies $1 \& 2\left(\nu_{2}>\nu_{1}\right)$. The function $f\left(\alpha_{12}\right)$ is the SID between the two frequencies. We have measured SIDs in several frequency bands: $1.4-5,5-8,8-20$ (or 8-22), and $22-43$. Depending on the frequency of the SZE experiment, we may need to apply Eq. 1 in several steps. For example, the RLF at $5 \mathrm{GHz}$ is obtained by extrapolating the $1.4 \mathrm{GHz}$ RLF with $f\left(\alpha_{1.4,5}\right)$, and can be used in conjunction with $f\left(\alpha_{5,8}\right)$ to obtain the $8 \mathrm{GHz}$ RLF. Convolving the latter with $f\left(\alpha_{8,20}\right)$ [or $f\left(\alpha_{8,22}\right)$ ] gives the RLF at $10-30 \mathrm{GHz}$ range. Finally, RLFs at higher frequencies (e.g., $145 \mathrm{GHz}$ ) are obtained by extrapolating the $22 \mathrm{GHz}$ RLF with $f\left(\alpha_{22,43}\right)$.

It is certainly preferable to utilize the full spectral shape from 5 to $43 \mathrm{GHz}$ of our sources for the extrapolation of the RLFs. We elect not to do so in the current analysis, as our determination of spectral shape below $22 \mathrm{GHz}$ may not be reliable. Instead, we treat the spectral indices at different frequency bands as independent, and extrapolate the RLFs in a piecewise fashion. This is justified given the lack of correlation of spectral indices in the radio two-color diagram (Fig. 3).

Ideally, one would extrapolate the RLF separately for the compact and extended components of radio sources. However, the $1.4 \mathrm{GHz}$ RLF presented by LM07 is based on fluxes from both the core and extended structures. Given that at low frequencies, the lobes usually dominate in flux over the cores (e.g., Fig. 2), the core-only RLF would have a smaller amplitude than the combined RLF. However, to determine the relative proportion of the core-only and the lobe-only RLFs, one needs to carefully examine all radio sources that contribute to the RLF, which is beyond the scope of the current analysis.

We note that the $\operatorname{SID}(1.4-4.85)$ derived from the NVSS/GB6 surveys should be representative for all sources with $\alpha_{1.4,4.85} \gtrsim-2$ (see Appendix), and therefore may result in an extrapolation of the $1.4 \mathrm{GHz}$ RLF (to $\sim 5 \mathrm{GHz}$ ) that appropriately takes into account the differences in the spectral shape of extended and compact sources. To further extrapolate to higher frequencies, we can use SIDs that are known to be biased towards positive and negative values of spectral indices, thus giving the (presumably) full range of possible RLFs. Our forecasts on the radio source contamination of

13 We note that radio-loud AGNs are known to reside in halos more massive than $\sim 10^{13} M_{\odot}$ or so (i.e., groups and cluster scale halos), based on their clustering properties (Mandelbaum et al. 2008; Wake et al. 2008). 
the SZE based on the RLFs will then reflect the incomplete knowledge of the source spectral shapes. To this end, for the four frequency ranges that we have determined the SIDs, we will employ a variety of SIDs to extrapolate the RLFs:

- 1.4-5 GHz: SID(1.4-4.85, NVSS/GB6).

- 5-8 GHz: SID(1.4-4.85, NVSS/GB6), SID(5-8, this work, point/core-like sources), or $\operatorname{SID}(5-8$, this work, all sources).

- 8-22 GHz: $\operatorname{SID}(8-22$, this work, all sources $)$ or $\operatorname{SID}(8-20$, AT20G).

- 22-43 GHz: SID(22-43, this work, point/core-like sources) or $\operatorname{SID}(22-43$, this work, all sources).

\subsection{Results}

As explained in the previous subsection, using various combinations of SIDs we estimate the possible range of the RLFs given the uncertainties in the spectral shape of the radio sources. The $z \sim 0$ extrapolated RLFs (within $r_{200}$ ) at 4 frequencies are shown as the shaded regions in Fig. 8. The bottom (top) panel shows the RLFs at $15 \& 30 \mathrm{GHz}(90 \& 145$ $\mathrm{GHz}$ ). At each frequency, the shaded region encloses the maximum and minimum of the RLFs resulted from the 12 SID combinations ( 6 for $15 \mathrm{GHz}$ ). For comparison, in both panels the solid curve is the $1.4 \mathrm{GHz}$ RLF.

Compared to a similar plot presented in LM07 (Fig. 13 therein), a dramatic change in the amplitudes of the extrapolated RLFs is seen. At $145 \mathrm{GHz}$, at the luminous end, the upper envelop of the RLF is about a factor of 60 less than that estimated in LM07. This is due to the combined effect of (1) the use of several SIDs in different frequency bands in the present analysis (so that the results are not strongly dependent on one single SID), and (2) that the SID used in LM07 may be biased to positive indices, as non-detections at $4.85 \mathrm{GHz}$ during the matching of sources between 1.4 and $4.85 \mathrm{GHz}$ were not properly taken into account (see Appendix for more discussion).

To check our extrapolation scheme, we show in Fig. 9 a comparison between our extrapolated and the observed RLFs at $28.5 \mathrm{GHz}$, using data from Coble et al. (2007). We restrict ourselves to sources in the 37 clusters in the Coble et al. (2007) sample which have redshifts in the range $0.1-0.3$, and for which X-ray observations are available, in order to match the selection criteria for our sources. Next, as the Coble et al. (2007) observations are made at the BIMA and OVRO arrays, we need to take account of the primary beam size of these arrays (6.6' and $4.2^{\prime}$, respectively). We therefore include only the 27 radio sources that fall inside a projected radius of $r_{2000} \approx 0.33 r_{200}$, as this radius roughly matches the FWHM beam of BIMA for clusters in the redshift range $0.1-0.3$. Using the source counts at $30 \mathrm{GHz}$ from Knox et al. (2004), we estimate that roughly 11 sources could be background objects. Given this uncertainty and the small number of sources, the RLF is not well determined. Furthermore, it is difficult to evaluate the impact of Coble et al.'s cluster selection on the resulting RLF (if anything, the amplitude of the RLF should be higher, because their cluster sample is selected against those hosting bright point sources). Nevertheless, it is reassuring to find that there is a general agreement between the data points and our extrapolation (shaded region).

We note that the redshift evolution of radio sources in clusters is an unresolved issue. Our sample is limited to

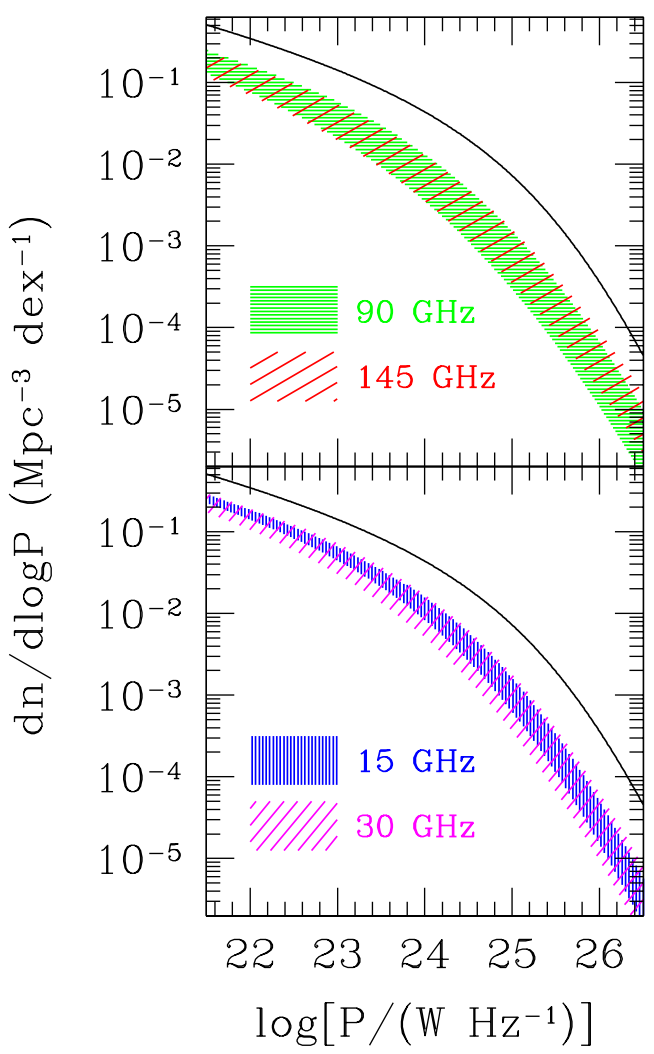

FIG. 8.- Radio luminosity function (RLF) of radio-loud AGNs at four frequencies. We transform the $1.4 \mathrm{GHz}$ cluster AGN RLF from LM07 (measured within the virial radius; solid line) to $15 \& 30 \mathrm{GHz}$ (bottom panel), and $90 \& 145 \mathrm{GHz}$ (top panel), using Eq. 1 To account for uncertainties in the spectral shapes of the sources, we use a variety of SIDs (see $\$ 8.1$ to extrapolate the RLF; the shaded regions enclose the probable range of the RLFs at these frequencies.

$z<0.25$, and currently there is no consensus as how radio galaxies evolve in massive halos (see LM07 for discussion). We have acquired $\mathrm{C}, \mathrm{X}$, and $\mathrm{K}$ band data for a sample of radio galaxies in $\sim 10$ intermediate-redshift clusters. Better constraints on the redshift evolution based on these new data will be presented in a future publication. In the current analysis, we will assume a pure density evolution of the form $\phi(z) \propto \phi(z=0)(1+z)^{\gamma}$, with $\gamma=1$, which corresponds to a factor of 2 increase of the density at $z \approx 1$. Such an evolution is derived from an analysis of the cluster radio source evolution from the Red sequence Cluster Survey (Roscioli \& Gladders 2008, in preparation; M. Gladders, 2008, private communication), and is much milder than what is assumed in LM07. The contamination of the SZE due to radio sources based on the present analysis is therefore much smaller when compared to the forecast presented in LM07.

Now, given the mass and redshift of a dark matter halo, we can estimate the degree of contamination as follows. For a halo, we denote the total fluxes from radio sources as $S_{A G N}$, and the SZE signal as $S_{S Z E}$. Using our Monte Carlo scheme to generate a large number of radio sources in massive halos, the fraction of halos for which $S_{A G N}$ is a significant fraction $q$ of $\left|S_{S Z E}\right|$ can be calculated. We consider two cases, $q=0.2$ and $q=1$, corresponding to $20 \%$ and $100 \%$ contamination. We show in Fig. 10 the resulting AGN contamination fraction (ACF) at $145 \mathrm{GHz}$, which is the proportion of the clusters 


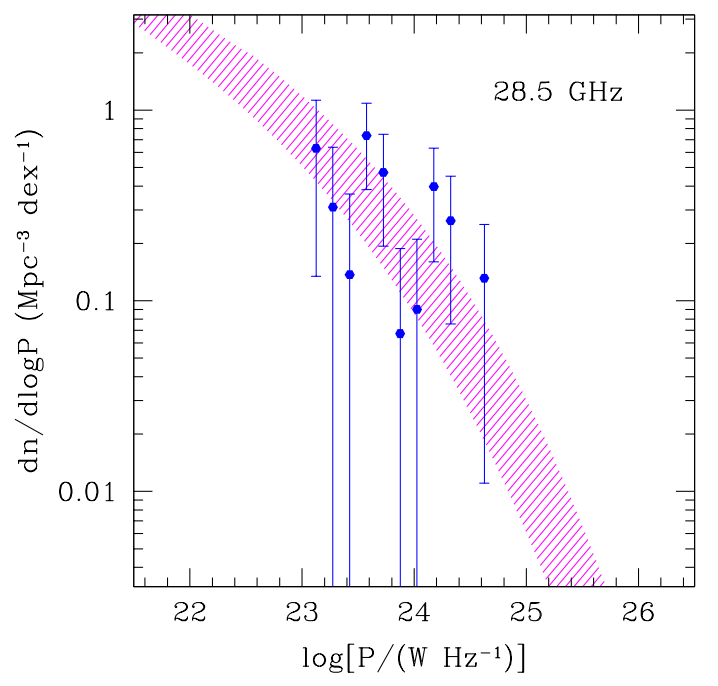

FIG. 9.- Comparison of our extrapolated RLF (shaded region) at 28.5 $\mathrm{GHz}$ with observations (points), for sources within $r_{2000}$ (about $1 / 3$ of the virial radius, $\left.r_{200}\right)$ in 37 clusters at $0.1<z<0.3$. The data is taken from Coble et al. (2007). Note that the extrapolated RLFs shown in Fig. 8 are for regions within $r_{200}$; for comparison with observations restricted to $r_{2000} \approx$ $r_{200} / 3$, we have scaled the $28.5 \mathrm{GHz}$ RLF within $r_{200}$ by a constant factor that takes into account the different spatial distribution of mass and radio sources [assuming the two components follow the Navarro et al. (1997) profile with concentration of 5 and 30 , respectively].

expected to host radio galaxies whose flux is $S_{A G N} \geq q\left|S_{S Z E}\right|$, as a function of cluster mass. The 3 panels show the results at $z=0.1,0.6$, and 1.1 (bottom to top). In each panel, the open points refer to the case of $q=0.2$, while the solid points show the $q=1$ ACF multiplied by a factor of $\mathbf{1 0}$ (for better presentation). Using different combinations of SIDs, we have constructed $12145 \mathrm{GHz}$ RLFs, resulting in a range of degree of contamination (at a given halo mass and redshift). While the points show the mean value of the contamination fractions, the error bars indicate the $1 \sigma$ range based on the 12 estimates.

The general trend shown in Fig. 10 is that ACF decreases as cluster mass and redshift increase. For $q=0.2$ contamination, the most affected clusters are those nearby, at $\sim 10 \%$ level for $M_{200}=10^{14} M_{\odot}$ clusters, reaching to $1-2 \%$ for $M_{200} \geq 10^{15} M_{\odot}$ ones. At $z=0.6$, an epoch close to the peak of cluster redshift distribution, the $q=0.2 \mathrm{ACF}$ is reduced to $<2 \%$ at $10^{14} M_{\odot}$, and becomes negligible towards high mass end. At $z=1.1$ the $q=0.2 \mathrm{ACF}$ is always at sub-percent level. Finally, the proportion of clusters that are affected by AGNs to $100 \%$ is a factor of $4-5$ smaller than the above estimates. For completeness, we note that about $0.4-4 \%(1-7 \%)$ of clusters in the mass range $10^{14}-10^{15} M_{\odot}$ at $z \sim 0.6$ may be contaminated to $10 \%$ (5\%) level (i.e., $q=0.1$ and 0.05 , respectively). At $z=1.1$, these values become $0.04-1 \%(0.2-2 \%)$.

We have provided a framework for estimating the abundance of radio-loud AGNs in halos. To better determine the impact of radio sources in SZE surveys, however, it is necessary to carry out mock observations that take into account the properties of the telescope and receiver system (e.g., angular resolution, sensitivity, frequency; see Sehgal et al. 2007), as well as the auxiliary observations (e.g., availability of multiwavelength data).

\section{SUMMARY AND FUTURE WORK}

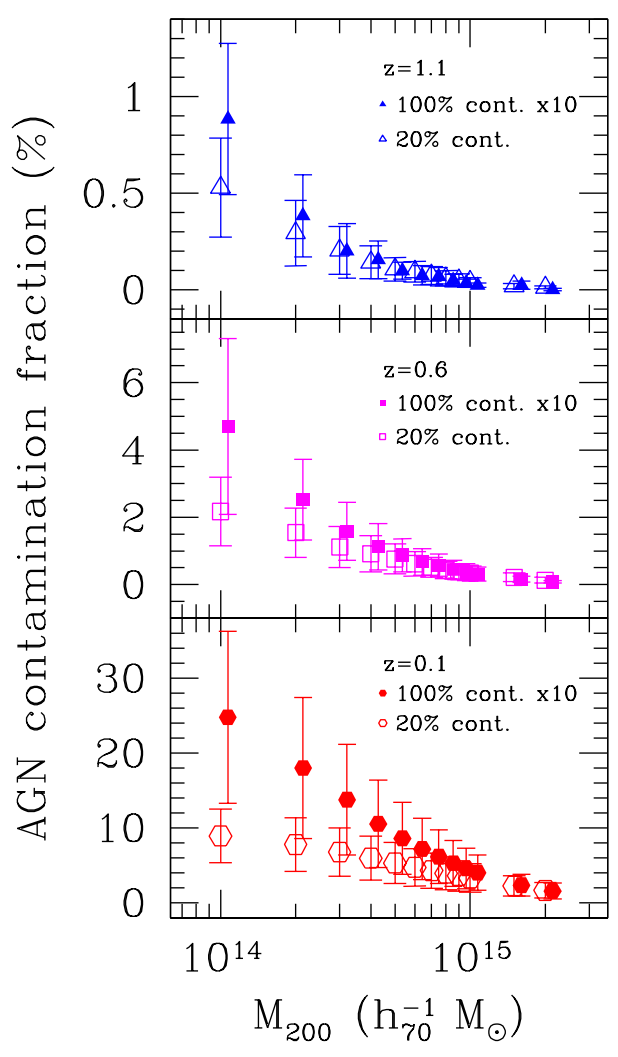

FIG. 10.- Fraction of clusters which host enough radio-loud AGNs such that their SZE signal measurements at $145 \mathrm{GHz}$ may be contaminated. We consider cases where the fluxes of the AGNs are at least a fraction $q$ of the SZE signal, $S_{A G N} \geq q\left|S_{S Z E}\right|$ (for the case where SZE signal is a temperature decrement), with $q=0.2$ (open symbols) and $q=1$ (solid symbols). Note the $q=1$ contamination fraction is multiplied by a factor of $\mathbf{1 0}$ for clarity of presentation. From bottom to top we examine clusters at $z=0.1,0.6$, and 1.1. A mild density evolution of the radio sources is assumed (corresponding to a factor 2 increase at $z=1$ compared to $z=0$ ). The contamination fraction is quite small (always $<10 \%$ ), and decreases with both cluster mass and redshift. The errorbars show the $1 \sigma$ range of possible degrees of contamination, reflecting our incomplete knowledge of the spectral shape of the radio sources.

We have presented a study of the spectral energy distribution of radio sources in a large sample of nearby clusters $(z<0.25)$. For 139 sources selected at $1.4 \mathrm{GHz}$ and spectroscopically confirmed to be members of the clusters, we use the VLA to measure the flux densities at 4.9, 8.5, 22, and 43 $\mathrm{GHz}(\mathrm{C}, \mathrm{X}, \mathrm{K}$, and Q bands) nearly simultaneously, and determine the distribution of the SED. Sources with extended morphology may be resolved out at high frequencies (i.e., reduction in flux due to the higher angular resolution of interferometer), making the determination of the spectral shape nontrivial. We have downgraded the resolution of our $43 \mathrm{GHz}$ images to match the resolution at $22 \mathrm{GHz}$, thus enabling reliable comparisons of fluxes at these two frequencies $(\$ 5.1)$; it is more difficult to match the resolution between the other frequency intervals, and therefore our measurements of the spectral indices involving the two lower frequencies (e.g., between 8.5 and $22 \mathrm{GHz}, \alpha_{X K}$, where $S \propto \nu^{\alpha}$ ) are lower limits. The flux measurement of point-like (or barely resolved) sources, or "cores" embedded in extended sources, on the other hand, is more straightforward.

Our main findings are the following:

1. For $\sim 70$ core/point-like sources that are detected in at 
least three frequencies, we study the distribution of the spectral shape via the "two-color" diagram (Fig. 3), and find that the spectral shape cannot be described by simple power-laws for the bulk of the sources. About $60 \%$ of sources have $\alpha_{K Q}>\alpha_{C X}$, indicating a flattening of the spectral shape above $8 \mathrm{GHz}$ or so; only $1 / 3$ of the sources have steep spectra in the entire range from 4.9 to $43 \mathrm{GHz}$.

2. We determine the spectral index distribution using survival statistics that take non-detections (upper limits) into account. The results are shown in Fig. 4 and Table 4. The compact sources are found to have "flatter" spectral shape than the extended sources.

3. The spectral indices do not correlate with properties of host galaxies or clusters, such as the color and luminosity of the galaxies, the radio luminosity at $1.4 \mathrm{GHz}$, the distance of the host galaxy to the cluster center, and the mass of the host clusters. This result agrees with previous studies, and suggests that the radio emission may be dominated by the small scale physics of the nucleus, rather than by the cluster environment.

4. In an attempt to estimate the contamination of the SZE signal due to radio point sources in cluster surveys, we make use of the spectral index distributions in several frequency bands to extrapolate the well-measured RLF at $1.4 \mathrm{GHz}$ to the frequencies employed by several on-going radio/millimeter wave experiments. As the extrapolation depends on the SIDs employed, we bracket the possible range of the predicted RLFs by using SIDs in intermediate frequency bands (e.g., $8-20 \mathrm{GHz}$ ) that are known to be biased in opposite ways. The amplitude of the resulting RLFs at $\nu \geq 30 \mathrm{GHz}$ is in general 5-20 times lower compared to that at $1.4 \mathrm{GHz}$. Under the assumption that the RLF follows a pure density evolution with redshift of the form $\phi(z) \propto \phi(z=0)(1+z)^{\gamma}$, such that the abundance of sources at $z=1$ is twice the local value, we find that the fraction of clusters that may be seriously affected by point sources is quite small; at the cluster mass scales close to the detection limits of the on-going surveys (e.g., $2-3 \times 10^{14} M_{\odot}$ ), and at the redshift where we expect the experiments to detect most of the clusters (i.e., $z \sim 0.6$ ), $\lesssim 2 \%$ of the clusters will be contaminated to $20 \%$ level or above (that is, the total fluxes from AGNs are at least $20 \%$ of the SZE signal).

There are two aspects that need to be improved for a better forecast within our analysis framework. Currently, the largest uncertainty in our modeling is the redshift evolution of cluster radio galaxies. If not properly accounted for, any unexpected evolution of the radio sources may be misinterpreted as changes in the cluster mass function, and cause errors in the determination of the properties of the dark energy. With our on-going VLA survey of cluster radio galaxies at intermediate redshift $(0.3 \leq z \leq 0.8)$, we plan to address this issue in a future publication.

In addition, in our forecast, it is implicitly assumed that the number of radio galaxies $\left(N_{R G}\right)$ a cluster can host is proportional to the cluster mass $\left(M_{200}\right)$. If, instead, $N_{R G} \propto M_{200}^{s}$ with $s<1$, we would overestimate the AGN contribution in high mass clusters. To check this assumption, one needs to determine the halo occupation distribution for radio galaxies. To this end, we have attempted to construct the halo occupation distribution of radio galaxies, using a large sample of radio galaxies in the local Universe (Lin et al. 2008, in preparation).

Mainly because of the very mild redshift evolution of the radio sources we adopt (which is based on the results from the RCS survey; Roscioli \& Gladders 2008, in preparation), we find that radio sources do not cause a substantial degree of contamination to the SZE signal. To control the systematics in the on-going and future SZE cluster surveys, it is thus crucial to understand the contamination due to the dusty IR sources. Rapid progress has been made on this regard (e.g., Righi et al. 2008; Fernandez-Conde et al. 2008). It would be important to perform an assessment of contamination due to both radio and IR sources within a single framework, which is the goal of our research in the near term.

We thank David Spergel, Jill Knapp, Anna Sajina, Joe Mohr, Tom Crawford, and Heinz Andernach for helpful comments and suggestions. We are grateful to Mike Gladders for sharing results on the evolution of radio sources in clusters prior to publication, and to an anonymous referee for constructive comments that have improved the paper. YTL thanks IH for constant encouragement and support. YTL acknowledges supports from the Princeton-Católica Fellowship, NSF PIRE grant OISE-0530095, FONDAP-Andes, and the World Premier International Research Center Initiative, MEXT, Japan. BP acknowledges support from NSF grant AST-0606975. A portion of this work was performed at JPL, under a contract with NASA.

The National Radio Astronomy Observatory is a facility of the National Science Foundation operated under cooperative agreement by Associated Universities, Inc.

Funding for the Sloan Digital Sky Survey (SDSS) and SDSS-II has been provided by the Alfred P. Sloan Foundation, the Participating Institutions, the National Science Foundation, the U.S. Department of Energy, the National Aeronautics and Space Administration, the Japanese Monbukagakusho, and the Max Planck Society, and the Higher Education Funding Council for England. The SDSS Web site is http://www.sdss.org/

The SDSS is managed by the Astrophysical Research Consortium for the Participating Institutions. The Participating Institutions are the American Museum of Natural History, Astrophysical Institute Potsdam, University of Basel, University of Cambridge, Case Western Reserve University, The University of Chicago, Drexel University, Fermilab, the Institute for Advanced Study, the Japan Participation Group, The Johns Hopkins University, the Joint Institute for Nuclear Astrophysics, the Kavli Institute for Particle Astrophysics and Cosmology, the Korean Scientist Group, the Chinese Academy of Sciences, Los Alamos National Laboratory, the Max-PlanckInstitute for Astronomy, the Max-Planck-Institute for Astrophysics, New Mexico State University, Ohio State University, University of Pittsburgh, University of Portsmouth, Princeton University, the United States Naval Observatory, and the University of Washington.

This research has made use of the NED and BAX databases, and the data products from the NVSS and GB6 surveys.

\section{APPENDIX}

\section{SPECTRAL INDEX DISTRIBUTIONS FROM NVSS/GB6 AND AT20G SURVEYS}

Here we describe the construction of the spectral index distributions, SID(1.4-5, NVSS/GB6) and SID(8-20, AT20G), shown in Fig. 7 
The NRAO VLA Sky Survey (Condon et al. 1998, NVSS) is a $1.4 \mathrm{GHz}$ survey covering the sky north of $\delta=-40^{\circ}$, with a resolution of $45^{\prime \prime}$. The nominal detection limit is $2.5 \mathrm{mJy}$. The Green Bank $4.85 \mathrm{GHz}$ (GB6) survey (Gregory et al. 1996) used NRAO's (former) $91 \mathrm{~m}$ telescope to survey the sky within $0^{\circ}<\delta<75^{\circ}$, with a resolution of $3.5^{\prime}$, and a detection threshold of $18 \mathrm{mJy}$. We first match the NVSS source catalog to the the spectroscopic sample of SDSS DR6 (with a conservative matching radius of $10^{\prime \prime}$ ), and limit the combined sample to $z<0.4$, as we are interested in the radio galaxies in the local Universe. We then cross correlate the NVSS/SDSS sample with GB6 (again using a conservative matching radius of $1^{\prime}$ ), keeping all unmatched NVSS/SDSS sources (for which we can derive upper limits on $\alpha_{L C}$ ). Because of the differences in the angular resolution of the two radio surveys, we further limit ourselves to NVSS sources for which there are no neighboring sources from NVSS within a radius of 4 ". This is to ensure that both surveys measure the "total" flux from the sources, and to avoid sources that might be blended in the lower resolution GB6 survey. Finally, to account for the differences in the detection limits, we set a high flux cut (100 mJy) for NVSS sources so that we can be sure to include all sources with $\alpha_{L C}>-1.4$. Of the resulting 292 NVSS sources, 9 are not detected in GB6, and we assign $18 \mathrm{mJy}$ as the upper limit in the $4.85 \mathrm{GHz}$ flux for these sources. The SID from this sample is shown in the lower panel in Fig. 7. We note our result is not sensitive to the flux cut applied to the NVSS sources, or on the requirement for the "isolatedness" of the sources. Neither setting the cut to $200 \mathrm{mJy}$ (so that we are complete for sources with $\alpha_{L C}>-1.9$ ) nor including sources with neighbors closer than $4^{\prime}$ changes the mean value of the SID beyond the one sigma level.

The Australia Telescope Compact Array is conducting a large survey at $20 \mathrm{GHz}$ (AT20G) that will eventually cover the sky south of $\delta=0^{\circ}$. A bright source catalog based on observations up to 2004 is reported by Sadler et al. (2006). We use the 114 sources stronger than $100 \mathrm{mJy}$ at $20 \mathrm{GHz}$ that are also detected at $8.6 \mathrm{GHz}$ to construct the SID, and show the result in the middle panel of Fig.7 Because the sources are selected at $20 \mathrm{GHz}$, the SID is biased towards positive values. Furthermore, the redshifts for the majority of the sources are not available, and thus the result may not be representative of the restframe 8-20 GHz SID. Nevertheless, this sample provides a distribution that is at the opposite extreme compared to that from our VLA observations, and therefore the two SIDs should bracket the true distribution.

\section{REFERENCES}

Abell, G. O. 1958, ApJS, 3, 211

AMI Collaboration, Barker, R., Biddulph, P., Bly, D., Boysen, R., Brown,

A., Clementson, C., Crofts, M., Culverhouse, T., Czeres, J., Dace, R.,

D'Alessandro, R., Doherty, P., Duffett-Smith, P.. Duggan, K., Ely, J.,

Felvus, M., Flynn, W., Geisbüsch, J., Grainge, K., Grainger, W., Hammet,

D., Hills, R., Hobson, M., Holler, C., Jilley, R., Jones, M. E., Kaneko, T.,

Kneissl, R., Lancaster, K., Lasenby, A., Marshall, P., Newton, F., Norris,

O., Northrop, I., Pooley, G., Quy, V., Saunders, R. D. E., Scaife, A.,

Schofield, J., Scott, P., Shaw, C., Taylor, A. C., Titterington, D., Velić, M.,

Waldram, E., West, S., Wood, B., Yassin, G., \& Zwart, J. 2006, MNRAS, 369, L1

Bolton, R. C., Cotter, G., Pooley, G. G., Riley, J. M., Waldram, E. M. Chandler, C. J., Mason, B. S., Pearson, T. J., \& Readhead, A. C. S. 2004, MNRAS, 354, 485

Carlstrom, J. E., Holder, G. P., \& Reese, E. D. 2002, ARA\&A, 40, 643

Coble, K., Bonamente, M., Carlstrom, J. E., Dawson, K., Hasler, N., Holzapfel, W., Joy, M., LaRoque, S., Marrone, D. P., \& Reese, E. D. 2007, AJ, 134, 897

Condon, J. J., Cotton, W. D., Greisen, E. W., Yin, Q. F., Perley, R. A., Taylor, G. B., \& Broderick, J. J. 1998, AJ, 115, 1693

Cooray, A. R., Grego, L., Holzapfel, W. L., Joy, M., \& Carlstrom, J. E. 1998, AJ, 115, 1388

de Young, D. S. 2002, The physics of extragalactic radio sources (University of Chicago Press, 2002.)

de Zotti, G., Ricci, R., Mesa, D., Silva, L., Mazzotta, P., Toffolatti, L., \& González-Nuevo, J. 2005, A\&A, 431, 893

Feigelson, E. D. \& Nelson, P. I. 1985, ApJ, 293, 192

Fernandez-Conde, N., Lagache, G., Puget, J.-L., \& Dole, H. 2008, A\&A, 481,885

Fowler, J. W., Niemack, M. D., Dicker, S. R., Aboobaker, A. M., Ade, P. A. R., Battistelli, E. S., Devlin, M. J., Fisher, R. P., Halpern, M., Hargrave, P. C., Hincks, A. D., Kaul, M., Klein, J., Lau, J. M., Limon, M., Marriage, T. A., Mauskopf, P. D., Page, L., Staggs, S. T., Swetz, D. S. Switzer, E. R., Thornton, R. J., \& Tucker, C. E. 2007, Appl. Opt., 46, 3444

Gregory, P. C., Scott, W. K., Douglas, K., \& Condon, J. J. 1996, ApJS, 103, 427

Isobe, T., Feigelson, E. D., \& Nelson, P. I. 1986, ApJ, 306, 490

Knox, L., Holder, G. P., \& Church, S. E. 2004, ApJ, 612, 96

Ledlow, M. J. \& Owen, F. N. 1995, AJ, 109, 853

-. 1996, AJ, 112, 9
Lima, M. \& Hu, W. 2005, Phys. Rev. D, 72, 043006

Lin, Y.-T. \& Mohr, J. J. 2003, ApJ, 582, 574

-. 2007, ApJS, 170, 71

Mandelbaum, R., Li, C., Kauffmann, G., \& White, S. D. M. 2008, MNRAS, submitted (arXiv:0806.4089)

Miller, N. A. \& Owen, F. N. 2001, ApJS, 134, 355

Morrison, G. E. \& Owen, F. N. 2003, AJ, 125, 506

Navarro, J. F., Frenk, C. S., \& White, S. D. M. 1997, ApJ, 490, 493

Owen, F. N. \& Ledlow, M. J. 1997, ApJS, 108, 41

Owen, F. N., Ledlow, M. J., \& Keel, W. C. 1995, AJ, 109, 14

Pierpaoli, E. \& Perna, R. 2004, MNRAS, 354, 1005

Reiprich, T. H. 2006, A\&A, 453, L39

Reiprich, T. H. \& Böhringer, H. 2002, ApJ, 567, 716

Righi, M., Hernández-Monteagudo, C., \& Sunyaev, R. A. 2008, A\&A, 478, 685

Ruhl, J., Ade, P. A. R., Carlstrom, J. E., Cho, H., Crawford, T., Dobbs, M., Greer, C. H., Halverson, N. w., Holzapfel, W. L., Lanting, T. M., Lee, A. T., Leitch, E. M., Leong, J., Lu, W., Lueker, M., Mehl, J., Meyer, S. S. Mohr, J. J., Padin, S., Plagge, T., Pryke, C., Runyan, M. C., Schwan, D., Sharp, M. K., Spieler, H., Staniszewski, Z., \& Stark, A. A. 2004, in Millimeter and Submillimeter Detectors for Astronomy II. Edited by Zmuidzinas, Jonas; Holland, Wayne; Withington, Stafford. Proceedings of the SPIE, Volume 5498 (2004), 11-29

Sadler, E. M., Ricci, R., Ekers, R. D., Ekers, J. A., Hancock, P. J., Jackson, C. A., Kesteven, M. J., Murphy, T., Phillips, C., Reinfrank, R. F. Staveley-Smith, L., Subrahmanyan, R., Walker, M. A., Wilson, W. E., \& de Zotti, G. 2006, MNRAS, 371, 898

Sadler, E. M., Ricci, R., Ekers, R. D., Sault, R. J., Jackson, C. A., \& de Zotti, G. 2008, MNRAS, 385, 1656

Sehgal, N., Trac, H., Huffenberger, K., \& Bode, P. 2007, ApJ, 664, 149

Staniszewski, Z., et al. 2008, ApJ, submitted (arXiv:0810.1578)

Sunyaev, R. A. \& Zel'dovich, Y. B. 1970, Ap\&SS, 7, 3

Toffolatti, L., Argueso Gomez, F., de Zotti, G., Mazzei, P., Franceschini, A., Danese, L., \& Burigana, C. 1998, MNRAS, 297, 117

Wake, D. A., Croom, S. M., Sadler, E. M., \& Johnston, H. M. 2008, MNRAS, accepted (arXiv:0810.1050)

White, M. \& Majumdar, S. 2004, ApJ, 602, 565

Zwart, A. C. J. T. L., et al. 2008, MNRAS, submitted (arXiv:0807.2469) 
TABLE 5

Cluster Radio Sources

\begin{tabular}{|c|c|c|c|c|c|c|c|c|c|c|c|c|c|c|}
\hline $\mathrm{Name}^{\mathrm{a}}$ & $\begin{array}{c}\text { RA } \\
(\mathrm{J} 2000)\end{array}$ & $\begin{array}{c}\text { Dec } \\
(\mathrm{J} 2000)\end{array}$ & Type $^{b}$ & $z^{\mathrm{c}}$ & $\begin{array}{c}f_{L}^{\mathrm{d}} \\
(\mathrm{mJy})\end{array}$ & $\underset{(\mathrm{mJy})}{f_{C}}$ & $\underset{(\mathrm{mJy})}{f_{X}}$ & $\underset{(\mathrm{mJy})}{f_{K}}$ & $\underset{(\mathrm{mJy})}{f_{Q}}$ & $\alpha_{C X}$ & $\alpha_{X K}$ & $\alpha_{K Q}$ & $\begin{array}{c}f_{90, C X} \mathrm{e} \\
(\mathrm{mJy})\end{array}$ & $\begin{array}{c}f_{90, K Q^{f}} \\
(\mathrm{mJy})\end{array}$ \\
\hline 0036-226B & 9.7842 & -22.3338 & $\mathrm{C}$ & 0.0654 & 129 & $74.33 \pm 0.73$ & $59.85 \pm 0.43$ & $39.12 \pm 0.95$ & $37.68 \pm 1.16$ & -0.39 & -0.44 & -0.06 & 23.77 & 36.12 \\
\hline $0037+209$ & 9.9397 & 21.2256 & -1 & 0622 & 143 & $10.43 \pm 2.05$ & $<0.66$ & $<1.77$ & $<1.49$ & $<-4.97$ & & & & \\
\hline $0037+292$ & 10.1180 & 29.5561 & $\mathrm{P} / \mathrm{C}$ & 0716 & 12 & $6.20 \pm 0.50$ & $2.91 \pm 0.32$ & $2.55 \pm 0.95$ & $<1.43$ & -1.36 & -0.14 & $<-0.88$ & 0.12 & \\
\hline $0039+211$ & 10.4230 & 21.4026 & $\mathrm{C}$ & 1030 & 670 & $101.70 \pm 2.20$ & $48.84 \pm 0.55$ & $24.70 \pm 0.65$ & $20.34 \pm 0.80$ & -1.32 & -0.70 & -0.30 & 2.15 & 16.35 \\
\hline 0039-095B & 10.4603 & -9.3031 & $\mathrm{C}$ & 0.0556 & 55 & $13.26 \pm 0.64$ & $7.04 \pm 0.24$ & $2.11 \pm 0.48$ & $<1.85$ & -1.14 & -1.24 & $<-0.20$ & 0.47 & $\ldots$ \\
\hline 0039-095A & 10.4509 & -9.2840 & -1 & 0.0556 & 48 & $20.98 \pm 2.09$ & $5.20 \pm 0.90$ & $<2.57$ & $<1.48$ & -2.51 & $<-0.72$ & & 0.01 & \\
\hline 0039-097 & 10.4592 & -9.4296 & $\mathrm{C}$ & 0.0556 & 82 & & $6.86 \pm 0.32$ & $4.10 \pm 0.48$ & $3.95 \pm 0.79$ & & -0.53 & -0.06 & & 3.79 \\
\hline $0043+201(1)$ & 11.6233 & 20.4671 & $\mathrm{C}$ & 0.1053 & 563 & $19.63 \pm 1.56$ & $14.93 \pm 0.50$ & $<1.68$ & $2.20 \pm 0.76$ & -0.49 & $<-2.24$ & & 4.65 & \\
\hline $0043+201(2)$ & 11.6222 & 20.4681 & $\mathrm{C}$ & 0.1053 & 563 & $19.63 \pm 1.56$ & $14.93 \pm 0.50$ & $10.63 \pm 0.48$ & $8.48 \pm 0.65$ & -0.49 & -0.35 & -0.35 & 4.65 & 6.58 \\
\hline $0046+011$ & 12.1665 & 1.4302 & $\mathrm{C}$ & 0.0632 & 68 & $25.57 \pm 0.88$ & $5.76 \pm 0.46$ & $2.63 \pm 0.57$ & $3.19 \pm 0.98$ & -2.68 & -0.80 & 0.29 & 0.01 & 3.96 \\
\hline $0047+241$ & 12.4245 & 24.4451 & $\mathrm{C}$ & 0.0818 & 200 & $25.23 \pm 1.08$ & $16.68 \pm 0.41$ & $7.01 \pm 0.53$ & $7.52 \pm 1.07$ & -0.75 & -0.89 & 0.11 & 2.86 & 8.14 \\
\hline $0047+242 \mathrm{~A}(1)$ & 12.4365 & 24.5003 & -1 & 0.0818 & 24 & $11.86 \pm 1.29$ & $5.62 \pm 0.57$ & $1.03 \pm 0.45$ & $<2.12$ & -1.35 & -1.74 & $<1.10$ & 0.23 & $\ldots$ \\
\hline $0047+242 \mathrm{~A}(2)$ & 12.4348 & 24.5008 & $\mathrm{C}$ & 0818 & 24 & $11.86 \pm 1.29$ & $4.82 \pm 0.49$ & $1.89 \pm 0.54$ & $<2.12$ & -1.62 & -0.96 & $<0.18$ & 0.20 & $\ldots$ \\
\hline $0050-220(1)$ & 13.3629 & -21.7503 & -1 & 0587 & 97 & $11.80 \pm 1.36$ & $6.78 \pm 0.55$ & & & -1.00 & & & 0.64 & \\
\hline $0050-220(2)$ & 13.3567 & -21.7366 & -1 & 0.0587 & 97 & $25.57 \pm 0.89$ & $13.94 \pm 0.51$ & $6.73 \pm 0.71$ & $3.21 \pm 0.76$ & -1.09 & -0.75 & -1.13 & 1.05 & 1.40 \\
\hline $0053+261 \mathrm{~A}(1)$ & .9624 & 26.4065 & -1 & 0.1971 & 1327 & $82.20 \pm 1.79$ & $21.74 \pm 0.68$ & $<2.40$ & $<2.21$ & -2.40 & $<-2.26$ & $\ldots$ & 0.08 & $\ldots$ \\
\hline $0053+261 \mathrm{~A}(2)$ & 9580 & 26.4131 & -1 & 0.1971 & 1327 & $81.37 \pm 2.06$ & $24.13 \pm 0.95$ & $<2.06$ & $<2.21$ & -2.19 & $<-2.52$ & $\ldots$ & 0.14 & $\ldots$ \\
\hline $0053-102 \mathrm{~B}$ & 9668 & -9.9847 & -1 & 0534 & 28 & $<1.95$ & $<0.45$ & $<2.40$ & $<1.85$ & & $\ldots$ & & . & $\ldots$ \\
\hline 0053-015 & .1068 & -1.2623 & $\mathrm{C}$ & 0.0444 & 1764 & $104.00 \pm 4.30$ & $44.33 \pm 1.16$ & $19.94 \pm 1.15$ & $18.98 \pm 0.75$ & -1.54 & -0.82 & -0.08 & 1.17 & 17.96 \\
\hline $0053-016$ & .0074 & -1.3430 & -1 & 0.0444 & 1095 & $282.00 \pm 8.00$ & $106.53 \pm-1.00$ & $7.77 \pm 1.74$ & $<1.62$ & -1.75 & -2.69 & $<-2.40$ & 1.68 & (1) \\
\hline $0100-221 \mathrm{~A}$ & 6880 & -21.9041 & $\mathrm{P}$ & 0.0566 & 138 & $6.11 \pm 0.57$ & $4.50 \pm 0.52$ & $<3.22$ & & -0.5 & $<-0.34$ & & 1.22 & $\ldots$ \\
\hline $0108+173$ & .7649 & 17.6521 & $\mathrm{P}$ & 0638 & 12 & $6.44 \pm 0.61$ & $3.51 \pm 0.2$ & $<1.96$ & $<1.90$ & -1. & $<-0.60$ & & 0.26 & \\
\hline $0110+152$ & .2483 & 15.4913 & $\mathrm{C}$ & 0444 & 719 & $31.50 \pm 0.60$ & $14.53 \pm 2.84$ & $4.97 \pm 0.65$ & $6.33 \pm 0.60$ & -1.3 & -1.10 & 0.37 & 0.54 & 8.30 \\
\hline $193(1)$ & .5867 & 19.5881 & $\mathrm{P}$ & 0544 & 20 & $11.88 \pm 0.61$ & & $5.25 \pm 0.46$ & $2.40 \pm 0$ & & -0 & -1.2 & 2.43 & 0.99 \\
\hline $0122+084$ & .2819 & 8.6994 & $\mathrm{P}$ & 0498 & 51 & $4.03 \pm 0.61$ & $3.32 \pm 0.32$ & $1.24 \pm 0.57$ & $2.42 \pm 0.47$ & -0 . & $-1 .($ & 1.02 & 1.45 & 5.13 \\
\hline$-016 \mathrm{~A}$ & .4345 & -1.3795 & $\mathrm{C}$ & 180 & 910 & $77.60 \pm 11.50$ & $20.02 \pm 1.67$ & $10.93 \pm-1.00$ & $6.84 \pm 0$ & -2 & -0 . & -0.7 & 0.06 & 4.04 \\
\hline & .5027 & -1.3451 & $\mathrm{P}$ & 0180 & 3270 & $7.53 \pm 9.16$ & $128.70 \pm 3.61$ & $121.70 \pm 1.40$ & $128.38 \pm 1.00$ & 0.3 & -0.0 & 0.08 & 276.79 & 136.33 \\
\hline-189 & .7266 & 19.2145 & $\mathrm{C}$ & 420 & 1345 & $310.00 \pm 4.26$ & \pm 1.91 & $24.98 \pm 1.23$ & $24.75 \pm 0.96$ & -2 . & -1 . & -0. & 0.18 & 24.49 \\
\hline $0139+07$ & .4977 & 7.6806 & $\mathrm{P}$ & 0616 & 43 & $6.27 \pm 1.04$ & $4.90 \pm 0.23$ & $2.07 \pm 0.64$ & $2.74 \pm 0.81$ & -0.4 & -0.8 & 0.43 & 1.71 & 3.76 \\
\hline $0139+$ & .5183 & 6506 & -1 & 0616 & 27 & $9.67 \pm 1.84$ & & $2.23 \pm 0.84$ & $<1.51$ & & -0. & $<-0.60$ & 0.03 & \\
\hline $0149+359(1)$ & 1932 & 36.1518 & -1 & 163 & 81 & $22.42 \pm 1.09$ & $10.78 \pm 0.47$ & $5.64 \pm 0.88$ & $5.40 \pm 0.64$ & -1.7 y & -0.66 & -0.07 & 0.48 & 5.14 \\
\hline $0149+359(2)$ & 1650 & 36.171 & $\mathrm{P}$ & 3 & 81 & $5.98 \pm 1.36$ & & & & -0 . & & & 3.89 & \\
\hline $0154+320(1)$ & 3257 & 32.2400 & -1 & 0894 & 372 & $91.00 \pm 2.00$ & $40.37 \pm 1.40$ & $<6.40$ & $<1.67$ & -1.4 & $<-1.89$ & & 1.26 & $\ldots$ \\
\hline $0154+320(2)$ & 3175 & 32.2465 & $\mathrm{C}$ & 0894 & 372 & $.00 \pm 2.00$ & $16.98 \pm 0.92$ & $3.15 \pm 1.08$ & $<1$ & & -1.73 & $<-1.04$ & 0.53 & \\
\hline $0304-122(1)$ & 46.7230 & -12.1091 & -1 & 0788 & 501 & 2.70 & $.07 \pm 1.89$ & $12.73 \pm 2.30$ & $2.35 \pm 1.08$ & -1. & -1.1 & -2.58 & 1.54 & 0.35 \\
\hline 0304-122(2) & 46.7184 & -12.1057 & -1 & 0788 & 501 & 2.00 & & $11.67 \pm 1.40$ & $6.56 \pm 1$ & & -1. & -0.88 & 2.79 & 3.43 \\
\hline $0304-122(3)$ & 46.7193 & -12.1061 & $\mathrm{C}$ & 0788 & 501 & $87.75 \pm 2.00$ & $.55 \pm 1.53$ & $4.93 \pm 1.44$ & $2.93 \pm 0.86$ & -1 . & -2.2 & -0.8 & 2.79 & 1.63 \\
\hline & 47.0678 & -23.5638 & -1 & & & $55.70 \pm 1.20$ & & $16.80 \pm 0.59$ & $14.15 \pm 1.06$ & & -0.7 & -0.2 & 3.97 & 11.67 \\
\hline$-134(1)$ & & -13.3701 & -1 & & 1160 & $106.00 \pm$ & 33.0 & $23.10 \pm 0.73$ & $16.96 \pm 0.74$ & -2 . & -0 . & -0.47 & 0.23 & 11.98 \\
\hline & & -20.4440 & $\mathrm{P}$ & 4 & 95 & & & $2.30 \pm 0.80$ & $<1.97$ & -2 & -0 . & $<-0.24$ & 0.02 & \\
\hline & & -20 & $\mathrm{C}$ & & 119 & & 17.37 & $10.24 \pm 0.63$ & $8.58 \pm 0.97$ & -1. & -0.54 & -0.27 & 0.25 & 7.03 \\
\hline & & & -1 & & 16 & & & $<2.12$ & $<1.46$ & -2. & $<-0.08$ & & 0.02 & \\
\hline & & & -1 & & 266 & $79.90 \pm 1.50$ & $38.21 \pm 0$ & $9.18 \pm 1.30$ & $6.17 \pm 0.99$ & -1. & -1 & -0.6 & 1.65 & 3.95 \\
\hline & & & -1 & & 2020 & $281.00 \pm 2.30$ & $140.29 \pm 2$ & $48.30 \pm 4.32$ & $19.62 \pm 1.80$ & -1.2 & -1 . & & 7.27 & 7.12 \\
\hline & .9480 & & $\mathrm{C}$ & & & & $.40 \pm 4.38$ & $23.90 \pm 6.30$ & $3.59 \pm 1$ & & & & & 0.43 \\
\hline $6+526(3)$ & & 5 & -1 & & 2020 & $497.10 \pm 2.80$ & $.00 \pm 3.00$ & $59.24 \pm 4.12$ & $35.33 \pm 2$ & -1 . & -1 & -0.7 & 16.43 & 19.75 \\
\hline $0836+290$ & 8160 & 8441 & $\mathrm{P}$ & & 1022 & $6.00 \pm 2.34$ & $153.60 \pm 0.91$ & $117.06 \pm 1.25$ & $95.22 \pm 1.18$ & & -0.2 & -0.3 & 143.78 & 75.48 \\
\hline & & & $\mathrm{C}$ & & & $.00 \pm 2.00$ & $11.57 \pm 1.45$ & $2.94 \pm 0.83$ & $1.43 \pm 0.64$ & -1 & -1.4 & -1.10 & 0.43 & 0.64 \\
\hline $0909+162(2)$ & .1408 & 15.9958 & -1 & 1 & 183 & $34.75 \pm 1.35$ & $.25 \pm 1.10$ & $<2.50$ & $<1.89$ & -1 . & $<-2.04$ & & 1.17 & \\
\hline & & & $\mathrm{P}$ & & 23 & $16.41 \pm 0.91$ & & $6.56 \pm 0.57$ & $7.56 \pm 0.63$ & & -0.48 & 0.22 & 1.51 & 8.87 \\
\hline $1058+107$ & 5.2392 & 10.5055 & $\mathrm{P}$ & & 24 & $9.96 \pm 0.39$ & $7.93 \pm 0.29$ & $4.85 \pm 0.68$ & $4.22 \pm 0.74$ & -0 . & -0 . & -0.21 & 3.02 & 3.61 \\
\hline $1108+289 \mathrm{~A}$ & 7.6990 & 28.6601 & $\mathrm{C}$ & 0321 & 34 & $9.50 \pm 1.30$ & $3.94 \pm 0.72$ & $2.55 \pm 0.57$ & $2.59 \pm 1.01$ & -1 & -0 . & 0.0 & 0.09 & 2.63 \\
\hline & 7.9145 & 40.8380 & -1 & 0.0794 & 771 & $235.00 \pm 2.00$ & $67.54 \pm 1.21$ & $16.30 \pm 2.17$ & $8.59 \pm 2.57$ & -2.25 & -1. & & 0.33 & 4.18 \\
\hline $1108+411(2)$ & 167.9144 & 40.8406 & $\mathrm{C}$ & 0.0794 & 771 & $235.00 \pm 2.00$ & $37.70 \pm 0.82$ & $13.37 \pm 1.65$ & $3.76 \pm 1.03$ & -3.30 & -1.06 & -1.94 & 0.19 & 0.90 \\
\hline $1108+411(3)$ & 167.9120 & 40.8391 & C & 0.0794 & 771 & $235.00 \pm 2.00$ & $71.01 \pm 1.46$ & $10.37 \pm 2.17$ & $\ldots$ & -2.16 & -1.97 & & 0.35 & \\
\hline
\end{tabular}


TABLE 5

\begin{tabular}{|c|c|c|c|c|c|c|c|c|c|c|c|c|c|c|}
\hline $1108+410 \mathrm{~A}$ & 167.9184 & 40.7853 & -1 & 0.0794 & 116 & $<4.35$ & $<1.21$ & $<2.37$ & $<1.46$ & & & & & \\
\hline 1108+410B & 167.9319 & 40.8210 & $\mathrm{P}$ & 0.0794 & 23 & $6.99 \pm 1.08$ & $5.27 \pm 0.79$ & $<2.44$ & $<1.49$ & -0.51 & $<-0.79$ & & 1.59 & \\
\hline $1113+295 \mathrm{~B}(1)$ & 169.1192 & 29.2860 & -1 & 0.0471 & 22 & $15.92 \pm 4.15$ & $<4.86$ & $4.14 \pm 1.05$ & $2.16 \pm 0.66$ & $<-2.14$ & $\ldots$ & -1.00 & & 1.04 \\
\hline $1113+295 B(2)$ & 169.0945 & 29.2523 & $\mathrm{P}$ & 0.0471 & 22 & $54.08 \pm 4.67$ & $31.78 \pm 4.06$ & $\ldots$ & $\ldots$ & -0.96 & $\ldots$ & $\ldots$ & 3.30 & \\
\hline $1113+295 \mathrm{C}$ & 169.1438 & 29.2547 & $\mathrm{C}$ & 0.0471 & 1888 & $289.90 \pm 6.10$ & $97.35 \pm 2.90$ & $35.72 \pm 1.95$ & $37.28 \pm 1.07$ & -1.97 & -1.03 & 0.07 & 0.93 & 39.12 \\
\hline $1129+562$ & 173.0960 & 55.9678 & -1 & 0.0531 & 39 & $8.22 \pm 0.49$ & $2.60 \pm 0.59$ & $<2.00$ & $<2.33$ & -2.07 & $<-0.27$ & $\ldots$ & 0.02 & \\
\hline $1130+148$ & 173.2561 & 14.5346 & $\mathrm{C}$ & 0.0834 & 167 & $13.68 \pm 0.41$ & $8.82 \pm 0.39$ & $3.42 \pm 0.76$ & $3.56 \pm 1.08$ & -0.79 & -0.97 & 0.06 & 1.36 & 3.73 \\
\hline $1130-037$ & 173.2713 & -4.0133 & $\mathrm{P}$ & 0.0484 & 791 & $158.51 \pm 2.10$ & $107.40 \pm 1.37$ & $62.13 \pm 0.86$ & & -0.70 & -0.56 & $\ldots$ & 20.45 & \\
\hline $1131+493$ & 173.4967 & 49.0622 & $\mathrm{C}$ & 0.0338 & 835 & $131.25 \pm 2.00$ & $91.44 \pm 1.32$ & $52.85 \pm 1.10$ & $41.84 \pm 0.92$ & -0.65 & -0.56 & -0.36 & 19.60 & 32.17 \\
\hline $1132+492$ & 173.6940 & 48.9562 & $\mathrm{C}$ & 0.0338 & 475 & $30.38 \pm 0.83$ & $31.86 \pm 0.74$ & $26.76 \pm 0.88$ & $18.59 \pm 0.86$ & 0.09 & -0.18 & -0.56 & 39.02 & 12.34 \\
\hline $1132+493$ & 173.7056 & 49.0779 & $\mathrm{P}$ & 0.0338 & 31 & $12.61 \pm 0.49$ & $7.15 \pm 0.43$ & $<2.45$ & $1.81 \pm 0.83$ & -1.02 & $<-1.10$ & & 0.64 & \\
\hline $1141+466(1)$ & 175.9146 & 46.3549 & $\mathrm{P}$ & 0.1162 & 814 & $149.40 \pm 0.50$ & $44.90 \pm 0.66$ & $3.47 \pm 1.04$ & $1.31 \pm 0.73$ & -2.17 & -2.63 & -1.49 & 0.27 & 0.44 \\
\hline $1141+466(2)$ & 175.9158 & 46.3564 & $\mathrm{C}$ & 0.1162 & 814 & $149.40 \pm 0.50$ & $66.15 \pm 0.62$ & $11.35 \pm 1.40$ & $0.95 \pm 0.56$ & -1.47 & -1.81 & -3.79 & 0.39 & 0.06 \\
\hline $1141+676$ & 176.1526 & 67.4060 & $\mathrm{P}$ & 0.1164 & 196 & $68.94 \pm 0.47$ & $37.75 \pm 0.51$ & $16.84 \pm 1.20$ & $10.48 \pm 0.95$ & -1.08 & -0.83 & -0.72 & 2.90 & 6.15 \\
\hline $1142+198$ & 176.2709 & 19.6064 & $\mathrm{C}$ & 0.0214 & 5450 & $723.75 \pm 9.55$ & $432.27 \pm 4.22$ & $215.48 \pm 2.70$ & $172.11 \pm 2.30$ & -0.93 & -0.71 & -0.34 & 48.08 & 133.66 \\
\hline $1153+736$ & 178.9965 & 73.4154 & $\mathrm{P}$ & 0.0836 & 64 & $27.66 \pm 0.59$ & $19.33 \pm 0.75$ & $3.01 \pm 1.09$ & $3.96 \pm 1.74$ & -0.65 & -1.91 & 0.42 & 4.20 & 5.39 \\
\hline $1155+266$ & 179.5839 & 26.3533 & $\mathrm{C}$ & 0.1120 & 880 & $13.80 \pm 6.70$ & $1.63 \pm 0.73$ & $6.92 \pm 0.93$ & $7.09 \pm 0.64$ & -3.85 & 1.48 & 0.04 & 0.00 & 7.29 \\
\hline $1159+583(1)$ & 180.5143 & 58.0337 & -1 & 0.1035 & 765 & $110.00 \pm 1.00$ & $53.15 \pm 0.88$ & $19.66 \pm 2.33$ & $8.70 \pm 1.70$ & -1.31 & -1.02 & -1.25 & 2.40 & 3.48 \\
\hline $1159+583(3)$ & 180.5203 & 58.0373 & -1 & 0.1035 & 765 & $124.00 \pm 1.10$ & $54.62 \pm 0.86$ & $23.74 \pm 3.00$ & $12.50 \pm 2.10$ & -1.48 & -0.85 & -0.98 & 1.66 & 6.07 \\
\hline $1201+282$ & 180.9028 & 27.9443 & $\mathrm{C}$ & 0.1390 & 215 & $2.93 \pm 0.31$ & $1.87 \pm 0.43$ & $4.94 \pm 0.86$ & $1.69 \pm 0.74$ & -0.81 & 1.00 & -1.64 & 0.28 & 0.51 \\
\hline $1201+026(1)$ & 181.0303 & 2.4099 & $\mathrm{C}$ & 0.0844 & 244 & $120.00 \pm 1.00$ & $29.82 \pm 1.32$ & $3.75 \pm 1.50$ & & -2.51 & -2.13 & & 0.26 & \\
\hline $1201+026(2)$ & 181.0264 & 2.4118 & $\mathrm{C}$ & 0.0844 & 244 & $120.00 \pm 1.00$ & $39.40 \pm 1.40$ & $8.11 \pm 0.96$ & $6.06 \pm 0.90$ & -2.01 & -1.62 & -0.45 & 0.34 & 4.37 \\
\hline $1201+026(3)$ & 181.2723 & 2.4135 & $\mathrm{C}$ & 0.0844 & 244 & $120.00 \pm 1.00$ & $25.65 \pm 1.06$ & & & -2.78 & & & 0.22 & \\
\hline $1207+722$ & 182.5808 & 71.9993 & $\mathrm{C}$ & 0.1226 & 256 & & $47.53 \pm 2.40$ & $6.56 \pm 2.09$ & $<2.58$ & & -2.03 & $<-1.43$ & & \\
\hline $1221+615(1)$ & 185.8762 & 61.2473 & -1 & 0.2308 & 321 & $69.15 \pm 0.57$ & $34.34 \pm 0.89$ & $12.50 \pm 1.49$ & $3.89 \pm 1.35$ & -1.26 & -1.04 & -1.78 & 1.74 & 1.05 \\
\hline $1221+615(2)$ & 185.8740 & 61.2521 & -1 & .2308 & 321 & $49.46 \pm 0.54$ & $24.78 \pm 0.69$ & $5.98 \pm 1.55$ & $<2.58$ & -1.24 & -1.46 & $<-1.28$ & 1.30 & \\
\hline $1224+091$ & 186.8264 & 8.8431 & $\mathrm{C}$ & 0.0896 & 48 & $<3.40$ & $5.05 \pm 0.68$ & $<2.39$ & $<2.91$ & & $<-0.77$ & & & \\
\hline $1225+636(1)$ & 186.9687 & 63.3840 & -1 & .1459 & 210 & $70.32 \pm 0.82$ & $19.53 \pm 0.65$ & $11.80 \pm 4.80$ & $<1.66$ & -2.31 & -0.52 & $<-3.00$ & 0.08 & \\
\hline $1225+636(2)$ & 186.9634 & 63.3848 & $\mathrm{C}$ & 0.1459 & 210 & $70.32 \pm 0.82$ & $22.41 \pm 0.63$ & $5.04 \pm 1.27$ & $5.47 \pm 1.00$ & -2.06 & -1.53 & 0.13 & 0.10 & 6.00 \\
\hline $1231+674$ & 188.3085 & 67.1289 & $\mathrm{C}$ & .1071 & 879 & $18.00 \pm 1.00$ & $11.00 \pm 1.33$ & $7.33 \pm 1.23$ & $4.43 \pm 0.84$ & -0.89 & -0.42 & -0.77 & 1.35 & 2.51 \\
\hline $1232+414(1)$ & 188.6250 & 41.1599 & $\mathrm{C}$ & 0.1908 & 689 & $<17.00$ & $19.51 \pm 2.40$ & $<2.67$ & $<1.39$ & . & $<-2.04$ & & & \\
\hline $1232+414(2)$ & 188.6142 & 41.1668 & -1 & 0.1908 & 689 & $105.30 \pm 1.50$ & $48.94 \pm 0.71$ & $19.85 \pm 2.50$ & $12.67 \pm 1.35$ & -1.38 & -0.93 & -0.69 & 1.87 & 7.65 \\
\hline $1233+169$ & 189.0338 & 16.6414 & $\mathrm{P}$ & 0.0784 & 630 & $189.00 \pm 2.50$ & $64.22 \pm 1.16$ & $33.21 \pm 0.99$ & $20.39 \pm 0.96$ & -1.94 & -0.68 & -0.75 & 0.65 & 11.78 \\
\hline $1233+168$ & 189.1079 & 16.5384 & $\mathrm{C}$ & 0.0784 & 1338 & $65.00 \pm 12.00$ & $11.13 \pm 2.40$ & $4.62 \pm 1.17$ & $3.28 \pm 0.90$ & -3.18 & -0.90 & -0.52 & 0.01 & 2.23 \\
\hline $1238+188$ & 190.2511 & 18.5537 & $\mathrm{C}$ & 0.0718 & 537 & $48.35 \pm 1.41$ & $18.32 \pm 0.80$ & $5.77 \pm 1.03$ & $4.75 \pm 0.91$ & -1.75 & -1.19 & -0.30 & 0.29 & 3.82 \\
\hline $1243+699$ & 191.4726 & 69.6582 & $\mathrm{C}$ & 0.2307 & 220 & $38.76 \pm 1.39$ & $6.66 \pm 0.60$ & $4.27 \pm 1.26$ & $0.80 \pm 0.43$ & -3.17 & -0.46 & -2.56 & 0.00 & 0.12 \\
\hline $1256+281$ & 194.8462 & 27.9112 & $\mathrm{C}$ & 0.0231 & 450 & $59.69 \pm 1.39$ & $37.62 \pm 1.53$ & $<2.56$ & $<1.85$ & -0.83 & $<-2.76$ & $\ldots$ & 5.26 & \\
\hline $1257+282(1)$ & 94.8964 & 27.9579 & -1 & 0.0231 & 215 & $39.83 \pm 0.89$ & $22.64 \pm 0.45$ & $7.83 \pm 1.94$ & $3.00 \pm-1.00$ & -1.02 & -1.09 & -1.47 & 2.04 & 1.02 \\
\hline $1257+282(2)$ & 194.9002 & 27.9610 & -1 & 0.0231 & 215 & $50.20 \pm 1.00$ & $23.19 \pm 0.47$ & $7.54 \pm 1.18$ & $2.82 \pm 0.71$ & -1.39 & -1.15 & -1.50 & 0.86 & 0.93 \\
\hline $1301+195$ & 5.9442 & 19.2715 & $\mathrm{P}$ & 0.06 & 74 & $22.51 \pm 0.28$ & $13.70 \pm 0.27$ & $6.20 \pm 0.75$ & $1.96 \pm 0.55$ & -0.89 & -0.81 & -1.76 & 1.65 & 0.54 \\
\hline 1300+677 & 195.6686 & 67.4780 & -1 & 0.1055 & 298 & $137.20 \pm 0.60$ & $87.48 \pm 0.77$ & $15.90 \pm 0.90$ & $<3.00$ & -0.81 & -1.75 & $<-2.55$ & 12.86 & $\ldots$ \\
\hline $1320+584(1)$ & 200.7321 & 58.1675 & -1 & 0.1932 & 325 & $78.62 \pm 1.02$ & $50.00 \pm 1.00$ & $13.94 \pm 1.33$ & $\ldots$ & -0.82 & -1.31 & $\ldots$ & 7.27 & \\
\hline $1320+584(2)$ & 200.7268 & 58.1711 & $\mathrm{C}$ & 0.1932 & 325 & $53.21 \pm 0.81$ & $27.87 \pm 0.67$ & $<2.85$ & $\ldots$ & -1.16 & $<-2.34$ & $\ldots$ & 1.77 & $\ldots$ \\
\hline $1333+412(1)$ & 203.8350 & 40.9999 & -1 & 0.2290 & 797 & $169.70 \pm 0.90$ & $63.05 \pm 0.62$ & $22.77 \pm 1.40$ & $\cdots$ & -1.78 & -1.04 & $\cdots$ & 0.93 & \\
\hline $1333+412(2)$ & 203.8320 & 41.0024 & -1 & 0.2290 & 797 & $161.30 \pm 0.90$ & $62.34 \pm 0.64$ & $18.44 \pm 1.21$ & $\ldots$ & -1.71 & -1.25 & $\ldots$ & 1.09 & \\
\hline $1339+266 \mathrm{~A}$ & 205.4552 & 26.3738 & -1 & 0.0724 & 40 & $15.90 \pm 0.64$ & $6.39 \pm 0.67$ & $<1.63$ & $<1.59$ & -1.64 & $<-1.40$ & $\ldots$ & 0.13 & \\
\hline $1339+266 \mathrm{~B}$ & 5.4606 & 26.3715 & -1 & 0.0724 & 287 & $91.95 \pm 0.61$ & $41.04 \pm 0.64$ & $1.87 \pm-1.00$ & $<1.59$ & -1.45 & -3.17 & $<-0.25$ & 1.32 & \\
\hline $1346+268 \mathrm{~A}$ & & 26.5928 & $\mathrm{P}$ & & 883 & $234.60 \pm 0.50$ & $110.74 \pm 0.84$ & $21.79 \pm 1.02$ & $12.24 \pm 0.67$ & -1.35 & -1.67 & -0.88 & 4.52 & 6.40 \\
\hline $1346+268 \mathrm{~B}$ & 7.2474 & 26.5594 & -1 & 22 & 35 & $10.75 \pm 0.67$ & $12.05 \pm 1.17$ & $4.14 \pm 0.96$ & $<1.50$ & 0.21 & -1 . & $<-1.55$ & 19.60 & \\
\hline $1415+084(1)$ & & 8.2084 & $\mathrm{C}$ & 0.05 & 331 & $29.83 \pm 1.66$ & $8.27 \pm 0.72$ & $3.60 \pm 0.59$ & $\ldots$ & -2.31 & -0.85 & $\ldots$ & 0.03 & . \\
\hline $1415+084(2)$ & 214.3826 & 8.2101 & $\mathrm{C}$ & 0.0570 & 331 & $29.83 \pm 1.66$ & $5.88 \pm 0.82$ & $<0.78$ & $\ldots$ & -2.93 & $<-2.07$ & $\ldots$ & 0.02 & \\
\hline $1418+253(1)$ & 215.1731 & 25.1499 & -1 & 0.0780 & 116 & $22.27 \pm 0.51$ & $9.45 \pm 0.75$ & $1.28 \pm 0.50$ & $1.70 \pm 0.71$ & -1.54 & -2.05 & 0.43 & 0.24 & 2.34 \\
\hline $1418+253(2)$ & 215.1747 & 25.1461 & -1 & 0.0780 & 116 & $<26.70$ & $6.63 \pm 0.76$ & $2.03 \pm 0.66$ & $3.76 \pm 0.84$ & & -1.21 & 0.94 & $\ldots$ & 7.53 \\
\hline $1418+253(3)$ & 215.1758 & 25.1438 & $\mathrm{C}$ & 0.0780 & 116 & $<26.70$ & $6.65 \pm 0.79$ & $2.15 \pm 0.80$ & $<1.56$ & -2.93 & -1.16 & $<-0.49$ & $\ldots$ & $\ldots$ \\
\hline $1418+253(4)$ & 215.1767 & 25.1408 & -1 & 0.0780 & 116 & $21.03 \pm 0.59$ & $6.36 \pm 0.65$ & $<1.74$ & $<1.84$ & -2.15 & $<-1.33$ & $\ldots$ & 0.04 & \\
\hline $1424+169(1)$ & 216.6422 & 16.7507 & -1 & 0.0528 & 97 & $9.55 \pm 0.58$ & $8.20 \pm 0.73$ & $\ldots$ & $\ldots$ & -0.27 & $\ldots$ & $\cdots$ & 4.28 & \\
\hline $1424+169(2)$ & 216.6313 & 16.7633 & -1 & 0.0528 & 97 & $7.94 \pm 0.70$ & $1.92 \pm 0.63$ & $\ldots$ & $\cdots$ & -2.56 & $\ldots$ & $\cdots$ & 0.00 & \\
\hline $1424+167(1)$ & 216.8221 & 16.5548 & -1 & 0.0528 & 103 & $11.93 \pm 0.57$ & $7.15 \pm 0.44$ & $4.75 \pm 1.32$ & $\ldots$ & -0.92 & -0.42 & $\ldots$ & 0.81 & \\
\hline
\end{tabular}


TABLE 5

Cluster Radio Sources

\begin{tabular}{|c|c|c|c|c|c|c|c|c|c|c|c|c|c|c|}
\hline $1435+249(1)$ & 219.3126 & 24.7591 & $\mathrm{C}$ & 0.0883 & 175 & $11.80 \pm 0.56$ & $9.21 \pm 0.39$ & $7.72 \pm 0.61$ & $5.77 \pm 0.58$ & -0.45 & -0.18 & -0.45 & 3.20 & 4.16 \\
\hline $1435+249(2)$ & 219.3168 & 24.7653 & -1 & 0.0883 & 175 & $11.69 \pm 0.88$ & $5.54 \pm 0.71$ & $<1.98$ & & -1.35 & $<-1.06$ & & 0.23 & \\
\hline $1435+250$ & 219.3200 & 24.8693 & $\mathrm{C}$ & 0.0883 & 206 & $23.03 \pm 0.73$ & $12.22 \pm 0.45$ & $6.53 \pm 1.10$ & $4.62 \pm 0.83$ & -1.14 & -0.64 & -0.53 & 0.82 & 3.12 \\
\hline $1433+553$ & 218.8688 & 55.1311 & $\mathrm{C}$ & 0.1396 & 447 & $73.92 \pm 1.02$ & $16.81 \pm 0.55$ & $7.95 \pm 0.83$ & $\ldots$ & -2.67 & -0.77 & $\ldots$ & 0.03 & $\ldots$ \\
\hline $1435+038(0)$ & 219.5993 & 3.6729 & $\mathrm{C}$ & 0.2240 & 801 & $103.16 \pm 0.78$ & $11.25 \pm 0.85$ & $<16.06$ & $\ldots$ & -3.99 & $<0.37$ & $\ldots$ & 0.41 & $\ldots$ \\
\hline $1435+038(1)$ & 219.5946 & 3.6713 & -1 & 0.2240 & 801 & $103.16 \pm 0.78$ & $47.29 \pm 0.54$ & $12.21 \pm 2.13$ & $\ldots$ & -1.40 & -1.39 & $\ldots$ & 1.70 & $\ldots$ \\
\hline $1435+038(2)$ & 219.5886 & 3.6702 & -1 & 0.2240 & 801 & $114.12 \pm 0.73$ & $55.45 \pm 0.51$ & $15.91 \pm 1.61$ & $\ldots$ & -1.30 & -1.28 & $\ldots$ & 2.56 & $\ldots$ \\
\hline $1435+038(4)$ & 219.5960 & 3.6714 & -1 & 0.2240 & 801 & $89.03 \pm 1.37$ & $<2.54$ & $<2.93$ & $\ldots$ & $<-6.41$ & $\ldots$ & $\ldots$ & & $\ldots$ \\
\hline $1452+188$ & 223.6312 & 18.6423 & $\mathrm{P}$ & 0.0579 & 38 & $11.99 \pm 0.23$ & $7.71 \pm 0.25$ & $2.11 \pm 0.56$ & $\ldots$ & -0.80 & -1.33 & $\ldots$ & 1.17 & $\ldots$ \\
\hline $1508+059(1)$ & 227.7339 & 5.7446 & -1 & 0.0767 & 489 & $60.51 \pm 0.46$ & $21.31 \pm 0.42$ & $4.07 \pm 1.03$ & $\ldots$ & -1.88 & -1.70 & $\ldots$ & 0.25 & $\ldots$ \\
\hline $1508+065(1)$ & 227.8630 & 6.3472 & -1 & 0.0817 & 552 & $127.65 \pm 0.72$ & $54.79 \pm 0.66$ & $18.02 \pm-1.00$ & $\ldots$ & -1.52 & -1.14 & $\ldots$ & 1.49 & $\cdots$ \\
\hline $1508+065(2)$ & 227.8584 & 6.3503 & -1 & 0.0817 & 552 & $127.44 \pm 0.70$ & $56.48 \pm 0.62$ & $15.35 \pm 2.17$ & $\cdots$ & -1.47 & -1.34 & $\ldots$ & 1.76 & $\ldots$ \\
\hline $1508+182(1)$ & 227.7893 & 18.0300 & -1 & 0.1163 & 346 & $74.34 \pm 0.88$ & $30.50 \pm 0.65$ & $<9.33$ & $\ldots$ & -1.60 & $<-1.22$ & $\cdots$ & 0.68 & $\cdots$ \\
\hline $1508+182(2)$ & 227.7847 & 18.0326 & -1 & 0.1163 & 346 & $68.61 \pm 0.96$ & $26.85 \pm 0.61$ & $6.90 \pm 1.51$ & $\cdots$ & -1.69 & -1.39 & $\ldots$ & 0.49 & $\ldots$ \\
\hline $1510+076(1)$ & 228.1405 & 7.4249 & -1 & 0.0451 & 17 & $10.59 \pm 1.54$ & $2.76 \pm 0.55$ & $<2.87$ & $\ldots$ & -2.42 & $<0.04$ & $\cdots$ & 0.01 & $\cdots$ \\
\hline $1510+076(2)$ & 228.1427 & 7.4318 & $\mathrm{C}$ & 0.0451 & 17 & $10.59 \pm 1.54$ & $1.97 \pm 0.45$ & $1.57 \pm 0.70$ & $\ldots$ & -3.03 & -0.23 & $\ldots$ & 0.01 & $\ldots$ \\
\hline $1514+072$ & 229.1854 & 7.0216 & $\mathrm{P}$ & 0.0348 & 5390 & $897.61 \pm 2.89$ & $691.40 \pm 2.70$ & $393.16 \pm 5.70$ & $\cdots$ & -0.47 & -0.58 & $\cdots$ & 227.36 & $\ldots$ \\
\hline $1520+087$ & 230.7719 & 8.6094 & -1 & 0.0355 & 13 & $5.59 \pm 0.47$ & $2.11 \pm 0.37$ & $<2.30$ & & -1.75 & $<0.09$ & & 0.03 & $\ldots$ \\
\hline $1525+290$ & 231.9350 & 28.9183 & $\mathrm{C}$ & 0.0656 & 224 & $88.69 \pm 0.64$ & $28.84 \pm 0.78$ & $5.70 \pm 0.84$ & $3.31 \pm 0.90$ & -2.02 & -1.66 & -0.83 & 0.24 & 1.80 \\
\hline $1530+282$ & 233.1860 & 28.0631 & $\mathrm{C}$ & 0.0734 & 352 & $133.24 \pm 1.03$ & $14.71 \pm 0.69$ & $4.87 \pm 0.82$ & $1.99 \pm 1.13$ & -3.97 & -1.13 & -1.37 & 0.00 & 0.73 \\
\hline $1531+312(1)$ & 33.3132 & 31.1285 & -1 & 0.0670 & 49 & $6.89 \pm 0.37$ & $2.81 \pm 0.32$ & $<2.17$ & $<1.61$ & -1.62 & $<-0.27$ & & 0.06 & $\ldots$ \\
\hline $1531+312(2)$ & 233.3137 & 31.1307 & $\mathrm{C}$ & 0.0670 & 49 & $6.89 \pm 0.37$ & $1.28 \pm 0.32$ & $<2.17$ & $<2.80$ & -3.03 & $<0.54$ & $\cdots$ & 0.03 & $\ldots$ \\
\hline $1531+312(3)$ & 233.3169 & 31.1331 & -1 & 0.0670 & 49 & $8.53 \pm 0.38$ & $4.39 \pm 0.45$ & $<2.17$ & $<2.25$ & -1.20 & $<-0.72$ & $\ldots$ & 0.26 & $\ldots$ \\
\hline $1555+356(2)$ & 239.4258 & 35.5076 & -1 & 0.1579 & 216 & $57.62 \pm 0.63$ & $14.60 \pm 0.41$ & $7.02 \pm 1.85$ & $3.03 \pm 0.90$ & -2.47 & -0.75 & -1.28 & 0.04 & 1.18 \\
\hline $1555+356(3)$ & 239.4267 & 35.5094 & $\mathrm{C}$ & 0.1579 & 216 & $23.70 \pm 0.44$ & $16.80 \pm 0.44$ & $5.38 \pm 0.91$ & $3.58 \pm 1.04$ & -0.62 & -1.17 & -0.62 & 0.83 & 2.26 \\
\hline $1556+274$ & 239.5585 & 27.2723 & -1 & 0.0896 & 130 & $30.38 \pm 0.41$ & $16.97 \pm 0.36$ & $7.97 \pm 1.59$ & $<1.92$ & -1.05 & -0.77 & $<-2.18$ & 1.42 & \\
\hline 1559+161(1) & 240.5703 & 15.9745 & -1 & 0.0354 & 17 & $6.83 \pm 0.36$ & $4.97 \pm 0.42$ & $<1.80$ & & -0.57 & $<-1.04$ & $\ldots$ & 1.28 & $\ldots$ \\
\hline $1602+178 \mathrm{~B}(1)$ & 241.2872 & 17.7314 & -1 & 0.0368 & 780 & $116.48 \pm 1.62$ & $76.02 \pm 2.36$ & $10.84 \pm 1.27$ & $\ldots$ & -0.77 & -2.00 & $\ldots$ & 12.34 & $\ldots$ \\
\hline $1602+178 \mathrm{~B}(1.5)$ & 241.2875 & 17.7299 & -1 & 0.0368 & 780 & & & $10.75 \pm 1.26$ & & & & . & & $\ldots$ \\
\hline $1602+178 \mathrm{~B}(2)$ & 241.2880 & 17.7270 & -1 & 0.0368 & 780 & $213.70 \pm 1.75$ & $139.80 \pm 2.39$ & $<4.73$ & & -0.76 & $<-3.47$ & & 22.92 & \\
\hline $1603+165$ & 241.3717 & 6.4357 & $\mathrm{C}$ & 0.0372 & 44 & $9.04 \pm 0.35$ & $4.78 \pm 0.33$ & $2.48 \pm 0.69$ & & -1.15 & -0.67 & $\ldots$ & 0.32 & $\ldots$ \\
\hline $1610+296(2)$ & 243.1456 & 29.4814 & $\mathrm{C}$ & 0.0320 & 119 & $38.00 \pm-1.00$ & $<0.59$ & $2.83 \pm 0.86$ & $<1.66$ & $<-7.50$ & $\ldots$ & $<-0.82$ & $\ldots$ & $\ldots$ \\
\hline $1626+396$ & 247.1594 & 39.5513 & -1 & 0.0299 & 3480 & $440.00 \pm-1.00$ & $185.00 \pm-1.00$ & $\ldots$ & $\ldots$ & -1.56 & $\ldots$ & $\ldots$ & 4.61 & $\ldots$ \\
\hline $1638+468$ & 250.0925 & 46.7131 & -1 & 0.2070 & 212 & $74.10 \pm 0.73$ & $44.70 \pm 0.84$ & & & -0.91 & $\ldots$ & $\ldots$ & 5.19 & $\ldots$ \\
\hline $1657+325 \mathrm{~A}$ & 254.7545 & 32.4941 & -1 & 0.0628 & 171 & $22.00 \pm-1.00$ & $1.18 \pm 0.30$ & $<3.28$ & $<1.82$ & -5.27 & $<1.05$ & $\ldots$ & 0.00 & $\ldots$ \\
\hline $1657+325 B$ & 254.7852 & 32.5000 & -1 & 0.0628 & 12 & & $<0.62$ & $<2.86$ & & & & & & \\
\hline $1707+344(1)$ & 7.4131 & 34.4283 & -1 & 0.0806 & 680 & $100.38 \pm 0.81$ & $56.81 \pm 1.53$ & $22.00 \pm 3.20$ & $13.90 \pm-1.00$ & -1.03 & -0.97 & -0.70 & 5.02 & 8.29 \\
\hline $1707+344(2)$ & 257.4096 & 34.4336 & -1 & 0.0806 & 680 & $108.22 \pm 0.74$ & $62.07 \pm 1.29$ & $23.00 \pm 2.50$ & $7.00 \pm 2.80$ & -1.00 & -1.02 & -1.82 & 5.81 & 1.84 \\
\hline & & 34.5120 & $\mathrm{P}$ & 0.0806 & 16 & $9.53 \pm 0.67$ & $5 \pm 0.81$ & $3.83 \pm 1.77$ & $>210$ & -1.18 & -0 . & $<-0.85$ & 0.30 & \\
\hline $1709+397 \mathrm{~B}$ & 7.7364 & 39.6926 & $\mathrm{C}$ & 0.0656 & 543 & $5 \pm 0.70$ & $60.28 \pm 0.75$ & $23.20 \pm 2.50$ & $12.13 \pm 2.50$ & -1.02 & -0.98 & -0.99 & 5.41 & 5.85 \\
\hline 1712 & 3.0974 & 64.03 & $\mathrm{C}$ & 0.08 & 290 & & 45. & $4.67 \pm 0.7$ & $<1.77$ & -1 & -2 & $<-1.48$ & 3.71 & $\ldots$ \\
\hline $1712+641(1)$ & 58.2699 & 64.1157 & -1 & 0.0808 & 66 & $26.93 \pm 0.99$ & $8.41 \pm 0.80$ & $3.59 \pm 1.23$ & $<1.87$ & -2.10 & -0.87 & $<-1.00$ & 0.06 & $\ldots$ \\
\hline $1712+641(2)$ & & 64.1177 & $\mathrm{C}$ & 0.0 & 66 & $3 \pm 0.99$ & & $1.28 \pm 0.41$ & $<1.87$ & -2 . & -1 . & $<0.58$ & 0.06 & $\ldots$ \\
\hline $1713+641(1)$ & 258.3701 & 64.0443 & -1 & 0.0808 & 250 & $85.10 \pm 0.66$ & $37.21 \pm 0.56$ & $9.33 \pm 1.44$ & $4.84 \pm 1.30$ & -1.49 & -1.42 & -1.00 & 1.10 & 2.31 \\
\hline $1713+641(2)$ & & 64.0457 & $\mathrm{C}$ & 0.08 & 250 & & $48.83 \pm 0.81$ & $7.26 \pm 0.79$ & $5.02 \pm 0.79$ & -1.00 & -1 . & -0.56 & 1.44 & 3.31 \\
\hline $1713+641(3)$ & 258.3735 & 64.0503 & -1 & 0.0808 & 250 & $70.30 \pm 0.65$ & $37.34 \pm 0.67$ & $4.26 \pm 0.88$ & $<1.86$ & -1.14 & -2.23 & $<-1.27$ & 2.52 & $\ldots$ \\
\hline+786 & 5.8676 & 78.63 & -1 & 0.05 & 157 & $42.81 \pm 0.69$ & $23.50 \pm 0.58$ & $5.98 \pm 1.11$ & $<1.78$ & -1.08 & -1.40 & $<-1.85$ & 1.82 & $\ldots$ \\
\hline+786 & 255.7604 & 78.5992 & -1 & 0.05 & 62 & $26.00 \pm-1.00$ & $6.91 \pm 0.73$ & $<2.30$ & $<1.83$ & -2.39 & $<-1.13$ & $\ldots$ & 0.02 & $\ldots$ \\
\hline $1706+787$ & 5.8668 & 78.6660 & $\mathrm{P}$ & 0.05 & 39 & $5.06 \pm 0.60$ & & & & -0.44 & $<-1.40$ & & 1.39 & \\
\hline $1703+787$ & 255.2176 & 78.6901 & -1 & 0.0581 & 10 & $2.14 \pm 0.45$ & $1.25 \pm 0.36$ & $<2.32$ & $<3.48$ & -0.97 & $<0.63$ & $\ldots$ & 0.13 & \\
\hline $1820+689$ & 274.9260 & 68.9476 & $\mathrm{C} / \mathrm{P}$ & 0.0880 & 801 & $83.27 \pm 1.61$ & $52.65 \pm 1.54$ & $31.11 \pm 1.12$ & $24.36 \pm 0.78$ & -0.83 & -0.54 & -0.37 & 7.47 & 18.50 \\
\hline $1826+747(1)$ & 276.2349 & 74.7308 & -1 & 0.1271 & 244 & $38.53 \pm 1.11$ & $21.63 \pm 0.71$ & $1.44 \pm 0.65$ & $1.85 \pm 0.65$ & -1.04 & -2.78 & 0.38 & 1.85 & 2.46 \\
\hline $1826+747(2)$ & 276.2153 & 74.7316 & -1 & 0.1271 & 244 & $44.98 \pm 1.27$ & $16.27 \pm 0.54$ & $3.68 \pm 1.30$ & $2.14 \pm 0.76$ & -1.83 & -1.53 & -0.83 & 0.21 & 1.16 \\
\hline $1849+702(1)$ & 282.3256 & 70.3535 & $\mathrm{C}$ & 0.0899 & 163 & $18.83 \pm 0.54$ & $14.47 \pm 0.34$ & $8.28 \pm 0.49$ & $9.48 \pm 0.65$ & -0.47 & -0 & 0.21 & 4.71 & 11.04 \\
\hline $1857+799$ & 3.4678 & 80.0474 & $\mathrm{P}$ & 0.2139 & 180 & $2.80 \pm 0.51$ & $2.66 \pm 0.27$ & $5.34 \pm 1.44$ & $2.61 \pm 0.60$ & -0.09 & 0.71 & -1.09 & 2.15 & 1.17 \\
\hline 2124-124(1) & 321.7414 & -12.2154 & -1 & 0.1760 & 251 & $96.09 \pm 1.10$ & $38.45 \pm 1.10$ & $<2.43$ & $<2.10$ & -1.65 & $<-2.83$ & & 0.78 & \\
\hline $2124-124(2)$ & 321.7384 & -12.2144 & $\mathrm{C}$ & 0.1760 & 251 & $96.09 \pm 1.10$ & $26.53 \pm 0.58$ & $8.96 \pm 1.13$ & $8.38 \pm 0.81$ & -2.32 & -1.11 & -0.10 & 0.54 & 7.77 \\
\hline $2142-202$ & 326.3143 & -19.9952 & -1 & 0.0576 & 351 & $2.45 \pm 1.27$ & $<0.70$ & $2.28 \pm 1.06$ & $<1.74$ & $<-2.26$ & $\ldots$ & $<-0.41$ & $\ldots$ & $\ldots$ \\
\hline
\end{tabular}


TABLE 5

\begin{tabular}{|c|c|c|c|c|c|c|c|c|c|c|c|c|c|c|}
\hline $2149-158 \mathrm{C}(1)$ & 327.9990 & -15.6384 & $\mathrm{C}$ & 0.0646 & 176 & $29.88 \pm 1.80$ & $12.76 \pm 1.03$ & $5.24 \pm 1.07$ & $1.83 \pm 0.75$ & -1.53 & -0.91 & -1.61 & 0.34 & 0.56 \\
\hline $2149-158 \mathrm{C}(2)$ & 327.9796 & -15.6263 & -1 & 0.0646 & 176 & $64.00 \pm 3.40$ & $14.67 \pm 1.70$ & $<2.93$ & $\ldots$ & -2.65 & $<-1.65$ & $\ldots$ & 0.03 & \\
\hline 2154-080A(1) & 329.2532 & -7.8474 & -1 & 0.0584 & 460 & $81.60 \pm 1.14$ & $51.47 \pm 2.65$ & $36.00 \pm-1.00$ & & -0.83 & -0.37 & & 7.22 & \\
\hline $2154-080 \mathrm{~A}(2)$ & 329.2569 & -7.8398 & $\mathrm{C}$ & 0.0584 & 460 & $81.20 \pm 1.13$ & $11.11 \pm 1.05$ & $5.45 \pm 0.53$ & $4.04 \pm 0.71$ & -3.58 & -0.73 & -0.46 & 0.00 & 2.88 \\
\hline $2154-080 \mathrm{~A}(3)$ & 329.2635 & -7.8362 & -1 & 0.0584 & 460 & $73.43 \pm 1.06$ & $46.53 \pm 2.41$ & $18.00 \pm-1.00$ & & -0.82 & -0.97 & 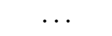 & 6.66 & \\
\hline $154-080 \mathrm{~B}$ & 329.3896 & -7.7943 & $\mathrm{C}$ & 0.0584 & 430 & $92.43 \pm 1.16$ & $13.88 \pm 0.71$ & $6.47 \pm 0.92$ & $5.67 \pm 0.96$ & -3.42 & -0.78 & -0.20 & 0.00 & 4.89 \\
\hline $228-087$ & 37.8701 & -8.4849 & $\mathrm{P}$ & 0.0810 & 107 & $10.89 \pm 0.67$ & $10.48 \pm 0.31$ & $6.39 \pm 0.72$ & $6.30 \pm 0.62$ & -0.07 & -0.51 & -0.02 & 8.89 & 6.20 \\
\hline 2229-086 & 37.9302 & -8.4088 & $\mathrm{C}$ & 0.0810 & 812 & $64.79 \pm 2.52$ & $33.38 \pm 1.13$ & $20.42 \pm 0.85$ & $16.84 \pm 0.70$ & -1.19 & -0.50 & -0.29 & 1.98 & 13.56 \\
\hline $247+106 B$ & 42.5818 & 10.9034 & -1 & 0.0768 & 14 & $2.44 \pm 0.24$ & $0.99 \pm 0.28$ & $3.07 \pm 1.08$ & $<1.11$ & -1.62 & 1.16 & $<-1.55$ & 0.02 & \\
\hline $321+164$ & 0.9762 & 16.6804 & -1 & 0.0416 & 46 & $20.40 \pm 0.57$ & $9.95 \pm 0.44$ & $6.96 \pm 0.10$ & $2.30 \pm-1.00$ & -1.29 & -0 . & -1.69 & 0.47 & 0.66 \\
\hline $2322+143 \mathrm{~A}(1)$ & 351.1339 & 14.6396 & $\mathrm{C}$ & 0.0421 & 187 & $65.59 \pm 1.36$ & $24.47 \pm 0.80$ & $6.75 \pm 0.92$ & $4.94 \pm 0.78$ & -1.78 & -1.32 & -0.48 & 0.37 & 3.47 \\
\hline $2322+143 \mathrm{~B}(1)$ & 1.1548 & 14.6425 & $\mathrm{C}$ & 0.04 & 76 & $25.85 \pm 0.95$ & $14.01 \pm 0.54$ & $6.84 \pm 1.05$ & $3.15 \pm 0.71$ & -1.10 & & -1.18 & 1.03 & 1.32 \\
\hline $2322-123$ & 51.3324 & -12.1241 & $\mathrm{P}$ & 0.0852 & 1699 & $415.59 \pm 0.91$ & $205.83 \pm 0.66$ & $59.58 \pm 1.22$ & $31.26 \pm 0.99$ & -1.27 & -1.27 & -0.99 & 10.31 & 15.13 \\
\hline $270(2)$ & & & -1 & 0.06 & 61 & & $<1.02$ & $<1.47$ & $<1.90$ & $<-3.97$ & 1.0? & . & & \\
\hline $2333+208(1)$ & 354.1270 & 21.1466 & $\mathrm{P}$ & 0.0569 & 55 & $11.41 \pm 0.33$ & $6.60 \pm 0.27$ & $4.30 \pm 0.62$ & $4.25 \pm 0.74$ & -0.99 & -0.44 & -0.02 & 0.64 & 4.19 \\
\hline $2333+208(3)$ & & & $\mathrm{P}$ & & 55 & & & $\ldots$ & & $\ldots$ & $\ldots$ & $\ldots$ & & \\
\hline $2335+267(1)$ & 354.6225 & 27.0314 & $\mathrm{C}$ & 0.0321 & 7650 & $267.48 \pm 15.60$ & $212.63 \pm 5.08$ & $139.12 \pm 2.16$ & $102.95 \pm 1.33$ & -0.41 & -0.44 & -0.46 & 79.97 & 73.37 \\
\hline $2335+267(2)$ & 54.6330 & 27.0244 & -1 & 0.0321 & 7650 & $316.87 \pm 22.30$ & $184.23 \pm 12.60$ & $<109.90$ & & -0.98 & $<-0.53$ & & 18.27 & \\
\hline $2348+058$ & 357.7107 & 6.1495 & $\mathrm{P}$ & 0.0556 & 50 & $8.49 \pm 0.29$ & $3.02 \pm 0.24$ & $5.28 \pm 1.88$ & $<1.77$ & -1.86 & 0.57 & $<-1.67$ & 0.04 & \\
\hline $2352+261(1)$ & 358.8519 & 26.4047 & $\mathrm{C}$ & 0.2404 & 311 & $3.89 \pm 0.84$ & $1.48 \pm 0.57$ & $1.91 \pm 0.82$ & $<3.25$ & -1.74 & 0.26 & $<0.81$ & 0.02 & \\
\hline $2352+261(3)$ & 358.8247 & 26.4162 & -1 & 0.2404 & 311 & $28.44 \pm 1.58$ & $12.30 \pm 0.85$ & & & -1.51 & & & 0.35 & \\
\hline
\end{tabular}

a Source name from Owen \& Ledlow [1997), which is based on B1950 positions; Parentheses denote multiple components

b Morphology of the sources: " -1 " denotes extended source; " $"$ " means core; "P" refers to point-like.

c Redshift taken from Owen \& Ledlow 1997).

d.4 GHz flux from Owen \& Ledlow [1997].

Predicted flux at $90 \mathrm{GHz}$ based on the fluxes at $\mathrm{C} \& \mathrm{X}$ bands and $\alpha_{C X}$. 